\title{
Buoyant gravity currents along a sloping bottom in a rotating fluid
}

\author{
By STEVEN J. LENTZ AND KARL R. HELFRICH \\ Department of Physical Oceanography, Woods Hole Oceanographic Institution, Woods Hole, \\ MA 02543, USA
}

(Received 21 September 2001 and in revised form 25 January 2002)

The dynamics of buoyant gravity currents in a rotating reference frame is a classical problem relevant to geophysical applications such as river water entering the ocean. However, existing scaling theories are limited to currents propagating along a vertical wall, a situation almost never realized in the ocean. A scaling theory is proposed for the structure (width and depth), nose speed and flow field characteristics of buoyant gravity currents over a sloping bottom as functions of the gravity current transport $Q$, density anomaly $g^{\prime}$, Coriolis frequency $f$, and bottom slope $\alpha$. The nose propagation speed is $c_{p} \sim c_{w} /\left(1+c_{w} / c_{\alpha}\right)$ and the width of the buoyant gravity current is $W_{p} \sim c_{w} / f\left(1+c_{w} / c_{\alpha}\right)$, where $c_{w}=\left(2 Q g^{\prime} f\right)^{1 / 4}$ is the nose propagation speed in the vertical wall limit (steep bottom slope) and $c_{\alpha}=\alpha g^{\prime} / f$ is the nose propagation speed in the slope-controlled limit (small bottom slope). The key non-dimensional parameter is $c_{w} / c_{\alpha}$, which indicates whether the bottom slope is steep enough to be considered a vertical wall $\left(c_{w} / c_{\alpha} \rightarrow 0\right)$ or approaches the slope-controlled limit $\left(c_{w} / c_{\alpha} \rightarrow \infty\right)$. The scaling theory compares well against a new set of laboratory experiments which span steep to gentle bottom slopes $\left(c_{w} / c_{\alpha}=0.11-13.1\right)$. Additionally, previous laboratory and numerical model results are reanalysed and shown to support the proposed scaling theory.

\section{Introduction}

The dynamics of gravity currents in a rotating reference frame is a classical problem that is relevant to a variety of geophysical applications (Simpson 1997). For example, river water discharging into saltier, and hence denser, ocean water turns cyclonically and forms a narrow buoyant gravity current that can flow hundreds of kilometres along the coast before dispersing (e.g. Munchow \& Garvine 1993; Hickey et al. 1998; Rennie, Largier \& Lentz 1999). The characteristics and dynamics of buoyant gravity currents along a vertical wall are relatively well understood from laboratory, theoretical and numerical model studies (Stern, Whitehead \& Hu 1982; Griffiths \& Hopfinger 1983; Kubokawa \& Hanawa 1984a,b; Griffiths 1986; Helfrich, Kuo $\&$ Pratt 1999). However, the relevance of these studies to the typical geophysical applications where the buoyant gravity current propagates along a sloping bottom is unclear. Previous laboratory (Whitehead \& Chapman 1986) and numerical modelling studies (Chao 1988; Chapman \& Lentz 1994; Kourafalou et al. 1996; Yankovsky \& Chapman 1997; Xing \& Davies 1999; Garvine 1999) suggest a sloping bottom has a profound influence on the characteristics and dynamics of buoyant gravity currents (hereafter referred to as simply gravity currents).

Studies have shown that gravity currents against a vertical wall in a rotating fluid are characterised by a blunt, turbulent nose region, followed by a region of geostrophic 

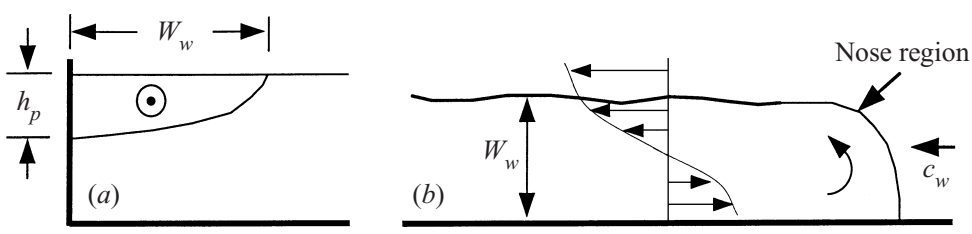

FIGURE 1. Schematic of a buoyant gravity current along a wall: $(a)$ cross-section and $(b)$ plan view. The circulation pattern in $(b)$ is in a coordinate frame moving with the nose and is based on Griffiths \& Hopfinger (1983).

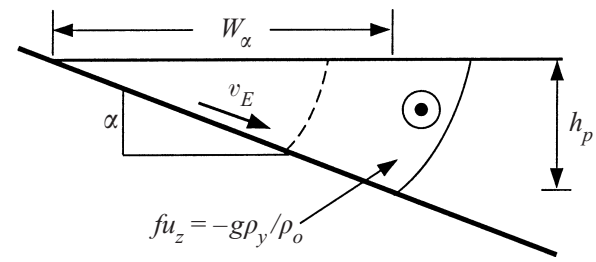

FIGURE 2. Schematic cross-section of a slope-controlled buoyant gravity current (following Chapman $\&$ Lentz 1994). Initially the foot of the front (dashed line) is advected offshore by the bottom Ekman transport until it reaches an equilibrium water depth (solid line) where the vertically sheared, geostrophic along-slope current is zero at the bottom.

flow (figure 1) (Stern et al. 1982; Griffiths \& Hopfinger 1983; Kubokawa \& Hanawa 1984a,b; Griffiths 1986). In the geostrophic region behind the nose, the thickness of the buoyant fluid along the wall is $h_{p}=\sqrt{2 Q f / g^{\prime}}$. The width $W_{w}=b_{1} L_{R}$ scales with the deformation radius $L_{R}=\sqrt{g^{\prime} h_{p}} / f$ and the propagation speed of the nose $c_{p}=b_{2} c_{w}$ scales with the internal wave speed $c_{w}=\sqrt{g^{\prime} h_{p}}$. Here the volume transport of the gravity current is $Q, f$ is the Coriolis frequency, $g^{\prime}=g \Delta \rho / \rho_{o}$ is reduced gravity, $g$ is the gravitational acceleration, $\Delta \rho=\left(\rho_{s}-\rho_{o}\right)$ is the density difference between the plume $\left(\rho_{s}\right)$ and ambient fluids $\left(\rho_{o}\right)$. The scaling coefficients $b_{1}$ and $b_{2}$ are found to be $O(1)$ from laboratory experiments (e.g. $b_{1}=0.42$ and $b_{2}=1.1$ from Stern et al. (1982); $b_{1}=0.6$ and $b_{2}=1.3$ from Griffiths \& Hopfinger (1983); $b_{1}=0.8$ and $b_{2}=1.0$ from Kubokawa \& Hanawa $\left.(1984 b)\right)$ ). In a reference frame moving with the nose, the flow component parallel to the wall is toward the nose near the wall (except for a narrow frictional boundary layer at the wall) and away from nose over the outer portion of the gravity current (figure $1 b$ ). In the laboratory studies, these flows rapidly develop instabilities which lead to lateral spreading of the current behind the nose.

A dynamical framework, similar to that for gravity currents along a wall, does not exist for the case of a sloping bottom. Whitehead \& Chapman (1986) found in a laboratory study that a gravity current on a sloping bottom was more stable, wider, and the nose propagated more slowly than a gravity current along a wall, but they did not offer a dynamical explanation for their results. Garvine (1999) found empirically from numerical model results that gravity current widths over a slope did not scale simply with the deformation radius based on the source conditions, but instead also depended on the bottom slope. A recent laboratory study by Avicola \& Huq (2002) also demonstrates the importance of bottom slope to the buoyant gravity current response.

Chapman \& Lentz (1994) and Yankovsky \& Chapman (1997) used numerical models to study a 'slope-controlled' (or 'bottom-trapped') gravity current, where most of the gravity current is in contact with a sloping bottom (figure 2). Slope-controlled 
currents have a different dynamical character than 'surface-trapped' gravity currents along a wall because bottom friction is an important component of the dynamics (Chapman \& Lentz 1994). The along-slope flow onshore of the foot of the front drives an offshore transport in the bottom Ekman layer that widens the gravity current until the vertically sheared, geostrophic flow at the density front separating the buoyant and ambient fluid is zero at the bottom. At this point, the offshore Ekman transport is zero because there is no longer a bottom stress. The gravity current reaches a geostrophic equilibrium and stops spreading offshore. Behind the nose region, the equilibrium gravity current transport is concentrated at the front and the flow is weak onshore of the front where the water is homogeneous. Yankovsky \& Chapman (1997) show that in equilibrium the depth where the front intersects the bottom is $h_{p}=\sqrt{2 Q f / g^{\prime}}$. This is the same as the gravity current depth along a vertical wall because in both cases the basic assumptions are that the flow is geostrophic and the flow is zero where the front intersects the bottom or the wall. For a constant bottom slope $\alpha$, the offshore distance to where the front intersects the bottom is $W_{\alpha}=h_{p} / \alpha$. The resulting steady gravity current can be significantly wider than the baroclinic deformation radius typical of a surface-trapped gravity current along a wall.

There is no criterion for evaluating whether the structure of a gravity current over an arbitrary slope will more closely resemble the surface-trapped (figure $1 a$ ) or slope-controlled (figure 2) case. Yankovsky \& Chapman (1997) examine this issue for the region near the source, but not for the coastal gravity current away from the source. Neither is there a theory for the nose propagation speed in the presence of a finite bottom slope. To address these problems, a scaling theory for gravity currents over a sloping bottom is proposed in $\S 2$. The theory builds on the previous results from studies of surface-trapped (along a wall) and slope-controlled gravity currents described above and provides estimates of the geometry (thickness and width), propagation speed and flow field characteristics of gravity currents along a slope as functions of $Q, g^{\prime}, f$ and $\alpha$. The theory recovers the earlier results for rotating gravity currents against a vertical wall in the limit of infinite bottom slope and the earlier results for slope-controlled gravity currents in the limit of a small bottom slope. The transition between these two limits is characterized by a single non-dimensional parameter. In $\S 3$ the proposed scaling is evaluated with a set of new laboratory experiments, and the results of previous laboratory experiments (Whitehead \& Chapman 1986) and numerical modelling studies (Chapman \& Lentz 1994; Yankovsky \& Chapman 1997; Garvine 1999). The results are discussed in $§ 4$ and summarized in $\S 5$.

\section{Scaling for buoyant gravity currents over a slope}

Consider a gravity current with transport $Q$ propagating along a boundary with a uniform bottom slope $\alpha$ (figure 3 shows two extremes). Assume the cross-slope momentum balance behind the nose is geostrophic, i.e. the Coriolis force $f u$ ( $u$ is the along-slope velocity) balances a cross-slope pressure gradient. Following Chapman \& Lentz (1994), assume that the offshore Ekman transport driven by the along-slope bottom stress moves the front separating the buoyant water from the ambient offshore water to an equilibrium depth where the near-bottom flow and hence bottom stress are zero. Once this equilibrium is reached, the depth at which the front intersects the bottom scales with (Yankovsky \& Chapman 1997)

$$
h_{p}=\left(\frac{2 Q f}{g^{\prime}}\right)^{1 / 2} \text {. }
$$


(a)

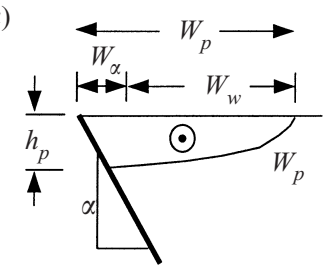

(b)

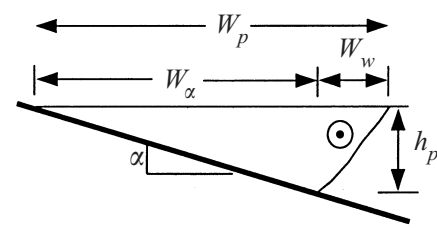

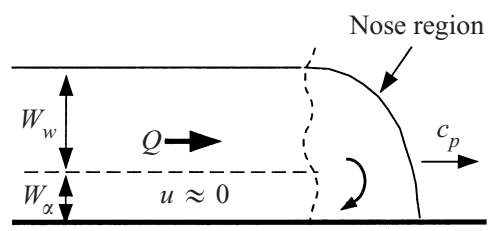

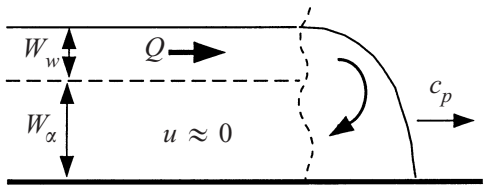

FIGURE 3. Schematic cross-sections and plan views of the buoyant gravity current geometry and circulation for $(a)$ surface-trapped $c_{w} / c_{\alpha} \ll 1$ and $(b)$ slope-controlled $c_{w} / c_{\alpha} \gg 1$ limits. The circulation patterns are in a stationary reference frame, in contrast to figure 1 .

As noted above, (1) is equally applicable to the limit of a vertical wall. The offshore distance to where the front intersects the bottom is

$$
W_{\alpha} \sim \frac{h_{p}}{\alpha}=\frac{c_{w}}{f} \frac{c_{w}}{c_{\alpha}}
$$

where

$$
c_{w}=\sqrt{g^{\prime} h_{p}}=\left(2 Q g^{\prime} f\right)^{1 / 4}
$$

is, to within an $O(1)$ constant, the propagation speed of a gravity current nose along a vertical wall. The parameter

$$
c_{\alpha}=\frac{\alpha g^{\prime}}{f}
$$

has units of velocity and is shown below to be the nose propagation speed scale in the limit of small bottom slope. Since the along-slope flow is geostrophic, assume that there is a geostrophic adjustment offshore of the foot of the front so that the distance from the foot of the front to the offshore edge of the gravity current scales with the deformation radius based on $h_{p}$, that is

$$
W_{w} \sim \frac{\sqrt{g^{\prime} h_{p}}}{f}=\frac{c_{w}}{f} .
$$

The total width of the gravity current at the surface is, from (2) and (5),

$$
W_{p}=W_{w}+W_{\alpha} \sim \frac{c_{w}}{f}\left(1+\frac{c_{w}}{c_{\alpha}}\right) .
$$

There are two implied coefficients on the right-hand-side of (6). However, for simplicity and clarity, the coefficient that multiplies $c_{w} / c_{\alpha}$ is assumed to be 1 . The evaluation of the scaling in $\S 3$ suggests this is a reasonable choice.

The propagation speed of the nose of the buoyancy current $c_{p}$ may be estimated by assuming that the ageostrophic nose region has a finite extent, the nose shape is steady as it propagates along the coast, and that there is minimal mixing of the gravity current fluid with the ambient fluid. Mixing and detrainment at the nose of gravity 
currents along a vertical wall is observed in laboratory experiments (Stern et al. 1982; Griffiths \& Hopfinger 1983; Kubokawa \& Hanawa 1984b). However, Whitehead \& Chapman (1986) found that mixing at the nose was nearly eliminated over a slope. With these assumptions, volume conservation at the nose of the buoyancy current implies $Q \Delta t=A c_{p} \Delta t$, where $A \sim \frac{1}{2} h_{p} W_{p}$ is the cross-sectional area of the plume (figure 3) and $\Delta t$ is a small time increment. Using (1) and (6),

$$
c_{p}=\frac{Q}{A} \sim \frac{c_{w}}{1+c_{w} / c_{\alpha}} .
$$

Thus, the propagation speed of the gravity current nose is always less than $c_{w}$ and tends to scale with the slower of the two propagation speeds $c_{w}$ or $c_{\alpha}$. Equations (1), (2), (6) and (7) give scales for the depth $h_{p}$, offshore distance to the foot of the front $W_{\alpha}$, width $W_{p}$, and nose propagation speed $c_{p}$ of a gravity current given $Q, g^{\prime}, \alpha$ and $f$.

The key non-dimensional parameter is the ratio

$$
\frac{c_{w}}{c_{\alpha}}=\left(\frac{2 Q f^{5}}{\alpha^{4} g^{\prime 3}}\right)^{1 / 4} \sim \frac{W_{\alpha}}{W_{w}} .
$$

Note that $c_{w} / c_{\alpha}$ is also the scale of the ratio of the isopycnal slope $\sim h_{p} / W_{w}$ to the bottom slope $\alpha$. The ratio $c_{w} / c_{\alpha}$ may be thought of as the inverse of a slope Burger number of the form $\alpha N / f$, with $N=\sqrt{g^{\prime} / h_{p}}$. If $c_{w} / c_{\alpha} \ll 1$, then $c_{p} \sim c_{w}, W_{p} \sim W_{w}$, and the buoyancy current is surface-trapped (figure $3 a$ ). In this limit the gravity current is independent of $\alpha$ and the scaling theory recovers the previous results for gravity currents along a wall. If $c_{w} / c_{\alpha} \gg 1$, then $c_{p} \sim c_{\alpha}, W_{p} \sim W_{\alpha}$, and the buoyancy current is slope-controlled (figure $3 b$ ). In this limit $c_{p} \sim \alpha g^{\prime} / f$ is independent of $Q$. The slope-controlled limit will occur for buoyancy currents with large transports (though the 1/4-power dependence is weak), at faster rotation rates (higher latitudes), smaller density anomalies, and smaller bottom slopes. The two limits for the nose speed, $c_{w}$ and $c_{\alpha}$, are analogous to the linear wave speeds of Kelvin waves and high-wavenumber, coastal-trapped topographic waves, respectively (e.g. LeBlond \& Mysak 1978). However, the dynamical connection is subtle since these gravity current flows are fundamentally nonlinear.

The character of the associated flow field varies with $c_{w} / c_{\alpha}$. Onshore of the foot of the front, the flow should be weak (Chapman \& Lentz 1994). This follows from the assumption that the along-slope flow is geostrophic, that in equilibrium the bottom stress is zero, and that cross-slope density gradients are small onshore of the front, so that there cannot be a substantial geostrophic vertical shear in this region. If the transport $Q$ is confined to the frontal region, then taking the area of the frontal region to be approximately $W_{w} h_{p} / 2$ and using (5) and (1), conservation of volume transport implies that the average velocity, $u_{p}$, is

$$
u_{p} \sim \frac{2 Q}{W_{w} h_{p}} \sim c_{w} .
$$

This provides a scale for the average velocity, but does not provide any insight into the structure of the flow within the frontal region (as seen for example in figure 1), which presumably depends on the shape of the interface, instabilities and mixing (Stern et al. 1982; Griffiths \& Hopfinger 1983). Thus, behind the gravity current nose the flow is divided into a region near the front moving at an average velocity $c_{w}$ and an onshore quiescent region (figure 3). Since from (7) and (9) $u_{p} / c_{p} \sim\left(1+c_{w} / c_{\alpha}\right) \geqslant 1$, 
the approaching flow must turn shoreward within the ageostrophic nose region and come to rest to fill the quiescent onshore region with buoyant water. In a frame propagating with the nose, the flow approaches the nose in the offshore region and flows back upstream near the coast. The flow structure provides a simple kinematic reason for the reduction in the nose propagation speed $c_{p}$ over a sloping bottom. The flow $u_{p}$ is confined near the front by geostrophy and is independent of the bottom slope. In the wall limit (figure 1), the entire gravity current is moving at an average flow speed of $u_{p}$. Since there is no quiescent onshore region, the nose propagates at the average flow speed, i.e. $c_{p}=u_{p}$. Over a sloping bottom, $c_{p}$ must be less than $u_{p}$ because as the flow approaches the nose, it takes time to fill in the quiescent onshore region. Both $h_{p}$ and $Q$ are independent of $\alpha$, so as the bottom slope decreases, the cross-sectional area of this quiescent onshore region increases, more time is required to fill in this region, and $c_{p}$ must decrease.

The scaling results are independent of the fluid viscosity or bottom stress, but the adjustment to an equilibrium state over a slope depends on the frictional, bottom Ekman layer. The time it takes for the gravity current to reach equilibrium is presumably related to two time scales: the time scale $f^{-1}$ for geostrophic adjustment of the front and the time it takes the foot of the front to move offshore a distance $W_{\alpha}$. The latter is approximately $W_{\alpha}$ divided by the average velocity in the bottom Ekman layer $v_{E}$. However, $v_{E}$ decreases as the front moves offshore because the near-bottom velocity and the bottom stress decrease as the front approaches its equilibrium position. Hence the rate at which the front moves offshore decreases with time. Assuming a constant kinematic viscosity, the offshore velocity in the bottom Ekman layer scales as $v_{E} \sim u_{b} / 2$, where $u_{b}$ is the near-bottom geostrophic velocity. For a narrow front with the along-slope velocity in thermal wind balance the velocity difference across the front is

$$
u_{p}-u_{b}(t) \sim \frac{g^{\prime} h(t)}{W(t) f},
$$

where $h(t)$ is the frontal depth which is increasing with time, $W(t) \sim \sqrt{g^{\prime} h(t)} / f, h / W$ is the slope of the interface, and we assume the velocity scale in the frontal region $u_{p}$ does not change with time. Since $u_{p} \sim c_{w}, u_{b} \rightarrow 0$ as $h(t) \rightarrow h_{p}$. The rate at which the foot of the front moves offshore is assumed to scale as the offshore Ekman velocity

$$
\frac{1}{\alpha} \frac{\mathrm{d} h(t)}{\mathrm{d} t} \sim v_{E} \sim \frac{u_{b}}{2} .
$$

Using (10) for $u_{b}$ in (11), using (9), (5) and (3), and integrating yields

$$
\hat{t} \sim-2 \sqrt{\hat{h}}-\ln (1+\hat{h}-2 \sqrt{\hat{h}})
$$

where $\hat{h}=h(t) / h_{p}, h_{p}$ is as defined in (1), $\hat{t}=t / t_{a d j}$ and

$$
t_{a d j}=\frac{2 W_{\alpha}}{c_{w}}=\frac{2 c_{w}}{f c_{\alpha}}
$$

is the adjustment time scale for the foot of the front to reach its equilibrium position. The equilibrium scaling is only valid for times longer than both $t_{a d j}$ and $f^{-1}$ after the initiation of the flow. Another natural time scale of the proposed scaling that will be used in the subsequent analysis is $t_{p}=W_{p} / c_{p}=\left(1+c_{w} / c_{\alpha}\right)^{2} / f$. The dynamical relevance of this time scale to interpretation of the laboratory experiments appears to be related to interfacial drag between the plume and ambient fluids and is discussed in $\S 4$. 


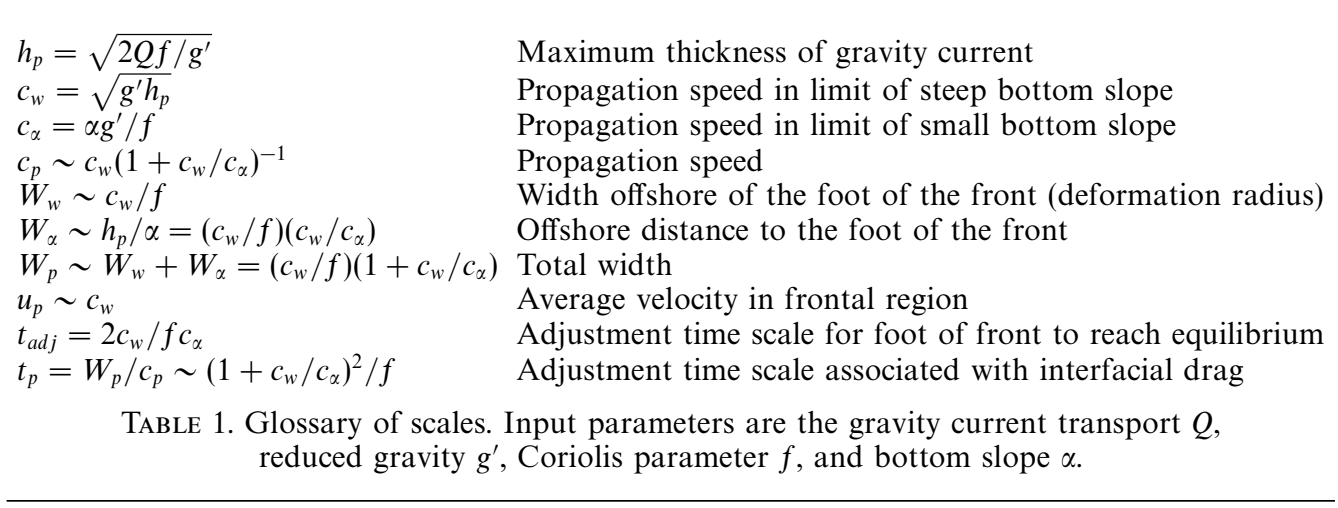

A summary of the scales introduced in this section is given in table 1 .

\section{Evaluation of the scaling}

The proposed scaling is evaluated using results from a set of new laboratory experiments. To further establish the general validity of the proposed scaling, the laboratory experiments of Whitehead \& Chapman (1986) and the results of previous numerical modelling studies (Chapman \& Lentz 1994; Yankovsky \& Chapman 1997; Garvine 1999) are also reanalysed and compared to the scaling theory. The evaluation has two objectives. The first is to test the qualitative predictions of the scaling theory, in particular the role of $c_{w} / c_{\alpha}$ in defining the importance of the slope and the structure of the flow field. The second is to determine the $O(1)$ constants implied in the scaling that are associated with, for example, assuming $W_{w}$ scales with the baroclinic Rossby radius of deformation, the neglect of mixing in the nose, and the over-simplified geometry used in estimating cross-sectional areas.

The determination of the $O(1)$ constants is complicated by the fact that the proposed scaling is based on the local $Q$ and $g^{\prime}$ of the equilibrium buoyancy current, but the source conditions are used to estimate the scales in the following evaluations. In general, $Q$ and $g^{\prime}$ in the equilibrium buoyancy current may not equal $Q$ and $g^{\prime}$ at the source for at least two reasons. First, entrainment of ambient fluid into the gravity current reduces $g^{\prime}$. Second, some of the source transport may not be carried by the gravity current, and instead is diverted into a growing recirculation zone near the source (e.g. Garvine 1999). This point is perhaps even more important in application of the results to the coastal ocean where studies have shown that a significant fraction of the source transport (e.g. river outflow) may either spread offshore as a growing bulge near the source or propagate upstream (in the opposite direction to the gravity current propagation) (Pichevin \& Nof 1997; Fong 1998; Garvine 1999, 2001). The fraction of the source transport carried by the gravity current decreases as the rotation rate decreases, until for $f=0$ the buoyant water spreads symmetrically away from the source and there is no buoyant coastal current (Garvine 1999). The fraction of the source transport carried by the gravity current also depends on the orientation of the source transport relative to the coast (Garvine 2001). Therefore comparisons include not only the $O(1)$ constants but also implicit relationships between values of $Q$ and $g^{\prime}$ at the source and in the gravity current. However, as discussed below, these effects are relatively small in our new laboratory experiments. 


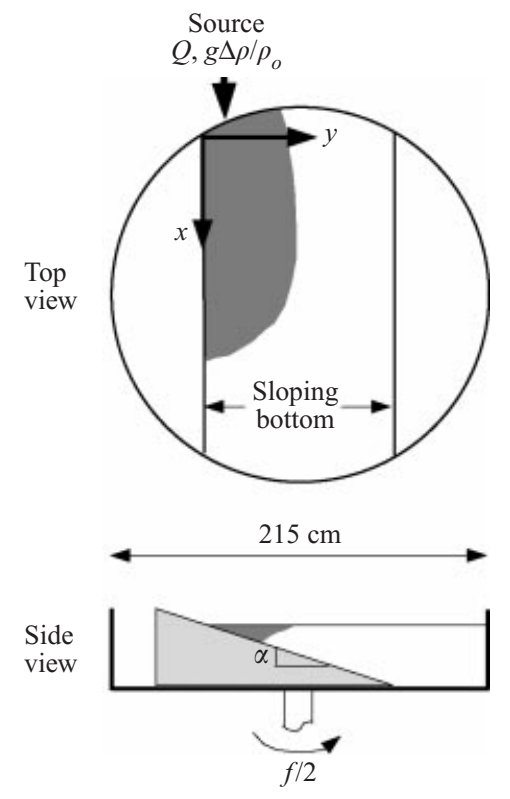

FIGURE 4. Schematic of the laboratory setup.

\subsection{Experimental setup and analysis procedures}

New laboratory experiments were conducted in a $2.1 \mathrm{~m}$ diameter cylindrical tank on the large rotating table in the Geophysical Fluid Dynamics Laboratory at Woods Hole Oceanographic Institution (figure 4). A straight section of uniformly sloping bottom (painted white) was placed in the tank. The tank was filled with ocean salt water $\left(\rho_{o} \approx 1.022 \mathrm{gm} \mathrm{cm}^{-3}\right)$ to a depth of $10 \mathrm{~cm}$ to $30 \mathrm{~cm}$ depending on the bottom slope. The densities were determined with a model DMA58 Anton Paar densitometer with an accuracy of $10^{-5} \mathrm{gm} \mathrm{cm}^{-3}$. Buoyant water (density $\rho_{s}$ ) was pumped into the counter-clockwise rotating (rotation rate $\Omega=f / 2$ ) tank at a fixed transport $Q$. The source was a $1.5 \mathrm{~cm}$ diameter pipe located over the slope, 4 to $23 \mathrm{~cm}$ offshore of the water's edge, with the discharge directed along the slope. A preliminary experiment showed that excessive mixing occurred near the source due to the jet-like outflow from the pipe. This mixing was largely eliminated by wrapping a piece of porous foam around the source to diffuse the outflow. After allowing the ambient fluid to reach solid body rotation the source was turned on and maintained until the gravity current encountered the far wall of the tank at the end of the slope region.

We conducted 28 experimental runs (table 2) divided into two groups designated LHa (runs 1-16) and LHb (runs 17-28). The source transport $Q=18.3 \mathrm{~cm}^{3} \mathrm{~s}^{-1}$ for LHa and $19.2 \mathrm{~cm}^{3} \mathrm{~s}^{-1}$ for LHb. The parameters $f\left(0.2-1 \mathrm{~s}^{-1}\right), \rho_{s}\left(0.998-1.020 \mathrm{gm} \mathrm{cm}^{-3}\right)$, and $\alpha(0.1$ or 0.29$)$ were varied to span a wide range of gravity current characteristics from surface-trapped $\left(c_{w} / c_{\alpha}=0.1\right)$ to slope-controlled $\left(c_{w} / c_{\alpha}=13\right)$. In LHa the buoyant water was dyed and the ambient water left clear to monitor the width of the gravity current at the surface $\left(W_{p}^{o b s}\right)$ and the nose propagation speed $\left(c_{p}^{o b s}\right)$. In LHb the ambient water was dyed and the buoyant water left clear to monitor the offshore distance to the foot of the front $\left(W_{\alpha}^{o b s}\right)$ and $c_{p}^{o b s}$. Each run was viewed from above with a co-rotating black and white video camera. Video images were digitized and stored directly on a computer at intervals of $1-4 \mathrm{~s}$ to form an image sequence of each run. The camera signal was also videotaped as a backup. During runs 3-16 


\begin{tabular}{|c|c|c|c|c|c|c|c|c|c|c|c|c|c|c|}
\hline Run & Q & $\alpha$ & $f$ & $g^{\prime}$ & $c_{w} / c_{\alpha}$ & $W_{p}$ & $W_{p}^{o b s}$ & $W_{\alpha}$ & $W_{\alpha}^{o b s}$ & $c_{p}$ & $c_{p}^{o b s}$ & $u_{p}$ & $u_{\max }$ & $y_{\max }$ \\
\hline 1 & 18.3 & 0.10 & 1.0 & 7.53 & 5.41 & 26.1 & 26.0 & 22.0 & 20.7 & 0.6 & 0.6 & 4.1 & - & - \\
\hline 2 & 18.3 & 0.10 & 1.0 & 21.75 & 2.44 & 18.3 & 19.4 & 13.0 & 10.6 & 1.5 & 1.4 & 5.3 & - & - \\
\hline 3 & 18.3 & 0.10 & 1.0 & 21.65 & 2.45 & 18.3 & 18.7 & 13.0 & 9.4 & 1.5 & 1.6 & 5.3 & 1.2 & 12.8 \\
\hline 4 & 18.3 & 0.10 & 1.0 & 2.32 & 13.08 & 42.7 & 39.6 & 39.7 & 18.6 & 0.2 & 0.3 & 3.0 & 5 & 21.4 \\
\hline 5 & 18.3 & 0.10 & 1.0 & 14.68 & 3.28 & 20.6 & 20.4 & 15.8 & 12.4 & 1.1 & 1.1 & 4.8 & 1.4 & 14.4 \\
\hline 6 & 18.3 & 0.10 & 0.5 & 22.12 & 1.01 & 18.1 & 18.3 & 9.1 & 8.6 & 2.2 & 2.0 & 4.5 & 1.7 & 12.6 \\
\hline 7 & 18.3 & 0.10 & 0.5 & 3.79 & 3.80 & 27.7 & 28.1 & 22.0 & 14.3 & 0.6 & 0.5 & 2.9 & 0.7 & 19.4 \\
\hline 8 & 18.3 & 0.10 & 0.5 & 13.97 & 1.43 & 19.4 & 20.8 & 11.4 & 9.1 & 1.6 & 1.5 & 4.0 & 1.4 & 13.6 \\
\hline 9 & 18.3 & 0.10 & 0.5 & 8.70 & 2.04 & 21.6 & 23.8 & 14.5 & 11.8 & 1.2 & 1.0 & 3.6 & 0.9 & 15.1 \\
\hline 10 & 18.3 & 0.10 & 0.5 & 2.11 & 5.91 & 34.4 & 39.9 & 29.5 & 18.4 & 0.4 & 0.3 & 2.5 & 0.6 & 17.2 \\
\hline 11 & 18.3 & 0.29 & 1.0 & 22.96 & 0.81 & 9.7 & 9.0 & 4.4 & 3.6 & 3.0 & 3.2 & 5.4 & 2.2 & 4.9 \\
\hline 12 & 18.3 & 0.29 & 1.0 & 3.10 & 3.63 & 15.1 & 15.3 & 11.8 & 6.8 & 0.7 & 1.0 & 3.3 & 1.3 & 10.6 \\
\hline 13 & 18.3 & 0.29 & 0.4 & 22.81 & 0.26 & 13.4 & 10.8 & 2.8 & 3.8 & 3.4 & 3.0 & 4.3 & 1.5 & 6.7 \\
\hline 14 & 18.3 & 0.29 & 0.2 & 22.53 & 0.11 & 19.9 & 17.4 & 2.0 & 3.6 & 3.2 & 2.4 & 3.6 & 1.4 & 2.0 \\
\hline 15 & 18.3 & 0.29 & 0.5 & 12.10 & 0.55 & 12.0 & 14.0 & 4.2 & 4.2 & 2.5 & 2.6 & 3.9 & 1.5 & 6.0 \\
\hline 16 & 18.3 & 0.29 & 0.2 & 12.17 & 0.17 & 18.0 & 18.5 & 2.7 & 4.0 & 2.6 & 2.0 & 3.1 & 1.3 & 5.4 \\
\hline 17 & 19.2 & 0.29 & 0.2 & 10.65 & 0.19 & 18.0 & - & 2.9 & 2.0 & 2.5 & 1.7 & 3.0 & - & - \\
\hline 18 & 19.2 & 0.29 & 1.0 & 4.41 & 2.82 & 13.8 & - & 10.2 & 7.8 & 0.9 & 1.5 & 3.6 & - & - \\
\hline 19 & 19.2 & 0.29 & 0.8 & 10.93 & 1.00 & 11.2 & - & 5.6 & 5.0 & 2.1 & 2.2 & 4.2 & - & - \\
\hline 20 & 19.2 & 0.29 & 1.0 & 22.35 & 0.84 & 9.9 & - & 4.5 & 3.7 & 2.9 & 3.0 & 5.4 & - & - \\
\hline 21 & 19.2 & 0.29 & 1.0 & 3.55 & 3.32 & 14.8 & - & 11.3 & 8.6 & 0.8 & 1.2 & 3.4 & - & - \\
\hline 22 & 19.2 & 0.29 & 0.5 & 11.13 & 0.52 & 12.6 & - & 4.3 & 3.2 & 2.5 & 2.9 & 3.7 & - & - \\
\hline 23 & 19.2 & 0.10 & 1.0 & 2.51 & 12.49 & 42.3 & - & 39.1 & 26.7 & 0.2 & 0.3 & 3.1 & - & - \\
\hline 24 & 19.2 & 0.10 & 1.0 & 2.41 & 12.86 & 43.0 & - & 39.9 & 21.3 & 0.2 & 0.4 & 3.1 & - & - \\
\hline 25 & 19.2 & 0.10 & 0.8 & 4.14 & 7.00 & 32.1 & - & 28.1 & 16.9 & 0.4 & 0.1 & 3.4 & - & - \\
\hline 26 & 19.2 & 0.10 & 0.7 & 8.33 & 3.25 & 23.5 & - & 18.0 & 15.2 & 0.9 & 1.0 & 3.9 & - & - \\
\hline 27 & 19.2 & 0.10 & 0.5 & 10.33 & 1.82 & 21.1 & - & 13.6 & 11.4 & 1.3 & 1.2 & 3.8 & - & - \\
\hline 28 & 19.2 & 0.10 & 0.2 & 15.43 & 0.43 & 23.6 & - & 7.1 & 4.9 & 2.3 & 1.8 & 3.3 & - & - \\
\hline
\end{tabular}

TABLE 2. Parameters and results for laboratory runs LHa (1-16) and LHb (17-28). All dimensional data in cgs units.

buoyant spheres (diameter $4 \mathrm{~mm}$ ) were placed in the gravity current to determine the characteristics of the near-surface velocity field.

The non-dimensional parameter $c_{w} / c_{\alpha}$ and scales for the widths $W_{p}$ and $W_{\alpha}$, the propagation speed $c_{p}$, and the average flow speed $u_{p}$ were estimated using (8), (6), (2), (7), (9) and the source values of $Q$ and $g^{\prime}, \alpha$ and $f$ (table 2). The observed propagation speed of the nose $c_{p}^{o b s}$ as a function of time was determined by differencing the along-slope positions of the leading edge of the nose in an image sequence and dividing by the time between images. The widths $W_{p}^{o b s}$ and $W_{\alpha}^{o b s}$ as functions of time were measured at a fixed location about halfway $(\approx 90 \mathrm{~cm}$ from the source) along the slope. For LHa, $W_{\alpha}^{\text {obs }}$ was estimated as the cross-slope location of the darkest portion (8-bit grey scale) of the gravity current in individual images assuming that the darkest value corresponds to the thickest point of the dyed gravity current. These estimates from LHa agree well with the more direct estimates of $W_{\alpha}^{o b s}$ from $\mathrm{LHb}$ (see figure 8). Buoyant sphere displacements were tracked from image to image to determine the surface water velocity within the gravity current. Average cross-slope profiles of along-slope velocity were determined within the region between 40 and $160 \mathrm{~cm}$ downstream of the source. This region was chosen to avoid complex flows near the source (discussed below) and at the end of the slope where the gravity current encounters the wall. The maximum of the average along-slope flow $u_{\max }$ 
and its cross-slope position $y_{\max }$ were determined and compared to $u_{p}$ and $W_{\alpha}$, respectively.

\section{Whitehead and Chapman (1986) experiments}

Observations from two sets of laboratory experiments by Whitehead \& Chapman (1986) are included in the evaluation of the scaling. The first set of experiments (designated WCa) were similar to ours. Buoyant fluid was pumped into an $89.8 \mathrm{~cm}$ diameter rotating tank at a constant rate $\left(Q=38 \mathrm{~cm}^{3} \mathrm{~s}^{-1}\right)$ along a vertical wall. The resulting gravity current flowed along the wall, encountered a sloping bottom $(\alpha=0.2)$, and then flowed along the slope. The values of $f$ and $\Delta \rho$ were varied in 12 different runs and the arrival times of the gravity current nose at $10 \mathrm{~cm}$ increments along the slope over a distance of $50 \mathrm{~cm}$ were recorded providing estimates of the propagation speed $c_{p}^{o b s}$. The widths $W_{p}$ and $W_{\alpha}$ were not recorded for these runs.

The second set of 19 runs (designated $\mathrm{WCb}$ ), conducted in a $2 \mathrm{~m}$ diameter tank, were similar to the first set except that the flow was generated by the release of a reservoir of buoyant fluid, i.e. a dam break. Again, the resulting gravity current first propagated along the vertical wall, encountered the slope, and then propagated along the slope. For this second set $f, \Delta \rho$ and the reservoir depth were varied. Because of the dam break initial condition the gravity current transport $Q$ was unknown. Therefore, the observed propagation speed along the wall $c_{w}^{o b s}$ is used to estimate $c_{w} / c_{\alpha}, W_{p}$ and $c_{p}$ by replacing $c_{w}$ with $c_{w}^{o b s}$ in (8), (6) and (7). (The use of $c_{w}=\sqrt{g^{\prime} H}$, where $H$ is the reservoir depth, yields similar results.) The width of the gravity current was measured by Whitehead \& Chapman 'soon' after the current reached the slope. Photographs suggest the gravity current width over the slope continued to increase with time. There were 17 runs in which both $W_{p}^{o b s}$ and $W_{p}$ could be estimated. (There were no reported measurements of $c_{w}^{o b s}$ in run 13 and $W_{p}^{o b s}$ in run 19 (see table 2 in Whitehead \& Chapman 1986).) Whitehead \& Chapman (1986) documented the position of the nose along the wall and slope as a function of time for 10 of the 19 runs (their figure 7). These data were used to estimate $c_{p}^{o b s}$ as a function of time after the gravity current began propagating along the slope. Thus, these experiments provide 17 estimates of $W_{p}^{o b s}$ (from WCb) and 22 estimates of $c_{p}^{o b s}$ (12 from WCa and 10 from $\mathrm{WCb}$ ). The parameter $c_{w} / c_{\alpha}$ ranged from 1.27 to 10.97 , with one run along a vertical wall.

\subsection{Evaluation of the scaling using laboratory results}

In each of our laboratory runs ( $\mathrm{LHa}$ and $\mathrm{LHb}$ ) a gravity current propagates along the slope. Examples of two runs with small and large values of $c_{w} / c_{\alpha}$ are shown in figure 5. Over the sloping bottom there was no obvious evidence of entrainment, except near the source, and little evidence for the development of frontal instabilities, as previously found by Whitehead \& Chapman (1986). This is in sharp contrast to the studies of gravity currents along a wall in which turbulent mixing and development of instabilities are common features (Stern et al. 1982; Griffiths \& Hopfinger 1983). The run in figure $5(a)$ has $c_{w} / c_{\alpha}=0.17$, well into the wall regime, yet shows no evidence of instability along the front. However, instabilities were observed once the gravity current reached the end of the slope region and began flowing along the vertical wall of the tank (not shown). Comparisons of the buoyant plume shape at different times (figure 6) show that the nose region propagates with essentially steady shape as assumed in the scaling development. For both extremes of $c_{w} / c_{\alpha}$, and for all the intermediate runs, the width and along-slope structure roughly scale as $W_{p}$. The gravity current width is approximately $W_{p}$ a distance of $1-2 W_{p}$ behind the nose. 
(a)

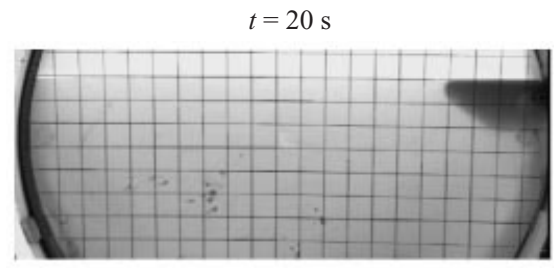

$t=60 \mathrm{~s}$

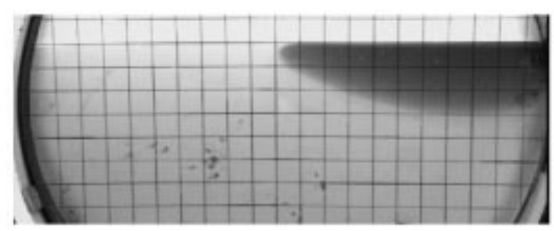

$t=150 \mathrm{~s}$

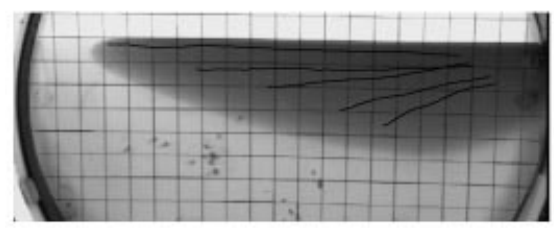

(b)

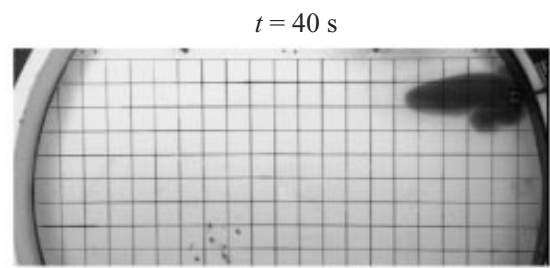

$t=120 \mathrm{~s}$

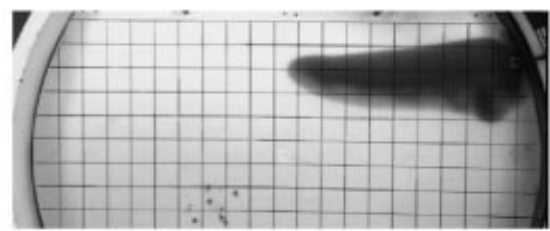

$t=320 \mathrm{~s}$

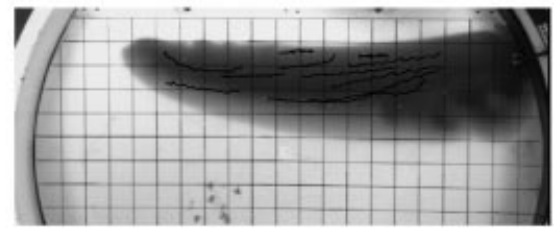

FiguRE 5. Examples of two laboratory runs, $(a)$ run $16 c_{w} / c_{\alpha}=0.17$ and $(b)$ run $4 c_{w} / c_{\alpha}=13$, at three times after the source was turned on. The lower panels include some of the surface particle tracks. The background grid has $10 \mathrm{~cm}$ spacing.

While it seems reasonable that the alongshore structure scales with $W_{p}$, this is not an explicit assumption of the scaling development.

Characteristics near the source varied. For $c_{w} / c_{\alpha} \geqslant 1$ a recirculating bulge often developed near the source (run 4, figure $5 b$ ). The presence and structure of the recirculating bulge depends in part on the location of the source. Runs 2 and 3 were nearly identical (table 2) except that the source was near the water's edge in run 2 and about $15 \mathrm{~cm}$ offshore in run 3 . A bulge developed near the source in run 3 but not in run 2. However, downstream of the source differences in the gravity current images and characteristics for the two runs were small. This suggests that the bulge caused little alteration of the gravity current transport or density. Water samples from the gravity current were taken during most runs to determine the density of the plume $\left(\rho_{p}\right)$. Densities in the gravity current were similar to the source values, $\left(\rho_{p}-\rho_{s}\right) /\left(\rho_{o}-\rho_{p}\right)<0.4$ and typically $<0.1$, indicating there was little mixing with the ambient water. The higher values of $\left(\rho_{p}-\rho_{s}\right) /\left(\rho_{o}-\rho_{p}\right)$ occurred for smaller values of $c_{w} / c_{\alpha}$ (i.e. surface-trapped gravity currents). Whether this has to do with conditions at the source, the proximity of the density samples to the foot of the front, or implies surface-trapped gravity currents entrain more than slope-controlled gravity currents is unclear. Thus, while the near-field region of the source shows interesting variations, it does not appear to have a substantial influence on the downstream characteristics 

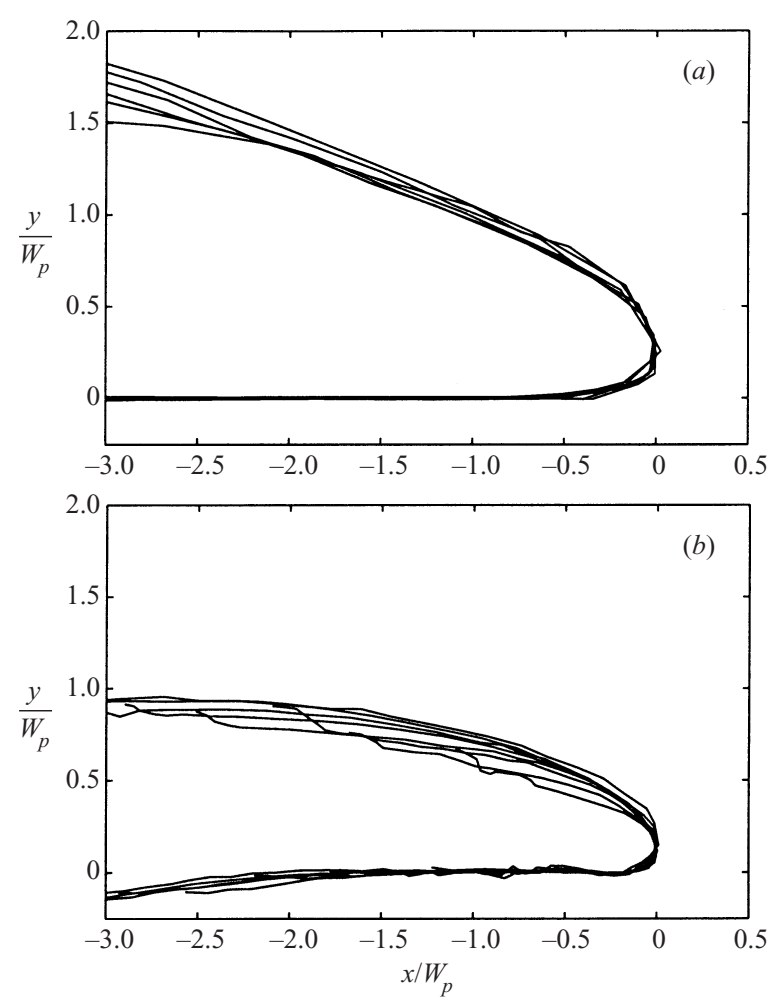

Figure 6. Outlines of plumes relative to the nose at several times during the two runs shown in figure $5,(a) c_{w} / c_{\alpha}=0.17$ at $t=38-138 \mathrm{~s}$ at intervals of $20 \mathrm{~s}$ and $(b) c_{w} / c_{\alpha}=13.1$ at $t=56-336 \mathrm{~s}$ at intervals of $40 \mathrm{~s}$. The $x$ (along-slope) and $y$ (cross-slope) dimensions are scaled by $W_{p}$. In $(b)$ the plume shapes have been rotated so that the $x$-axis is parallel to the shore near the nose to reduce effects of the shoreline curvature at this high rotation rate.

of the gravity current. In the analysis below we focus on the region downstream of the source.

\section{Bottom width}

Since $h_{p}$ and $W_{\alpha}$ are simply related by (2) given $\alpha$, this comparison focuses on measurements of $W_{\alpha}$. Values of $W_{\alpha}^{\text {obs }}$ as functions of time $t$ since the nose reached the measurement position increase rapidly initially, then increase more gradually, approaching a constant value ranging from 2 to $25 \mathrm{~cm}$ depending on the particular run (figure $7 a$ ). (Runs LHb are shown in figure 7 because they provide clearer estimates of $W_{\alpha}^{\text {obs }}$ than runs LHa, which show the same time dependence but are noisier.) Normalizing time by $t_{a d j}$, the time scale for the foot of the front to move offshore to its equilibrium position, and $W_{\alpha}^{\text {obs }}$ by $W_{\alpha}$ tends to collapse the observations (figure $7 b$ ). The normalized widths approach roughly constant values between 0.7 and 0.9 for $t>(10-30) t_{a d j}$. Runs with $c_{w} / c_{\alpha}>7$ were too short $\left(t / t_{a d j}<30\right)$ to have reached steady state. For these runs $t_{a d j}$ is relatively long $(15-25 \mathrm{~s})$ because these gravity currents are wide at the bottom and consequently it takes longer for the foot of the front to move offshore to the equilibrium position. The observed time dependence of the normalized widths is similar to the theoretical time dependence (dashed line figure 7) given by (12), though the normalized widths approach a value that is less than 1. 

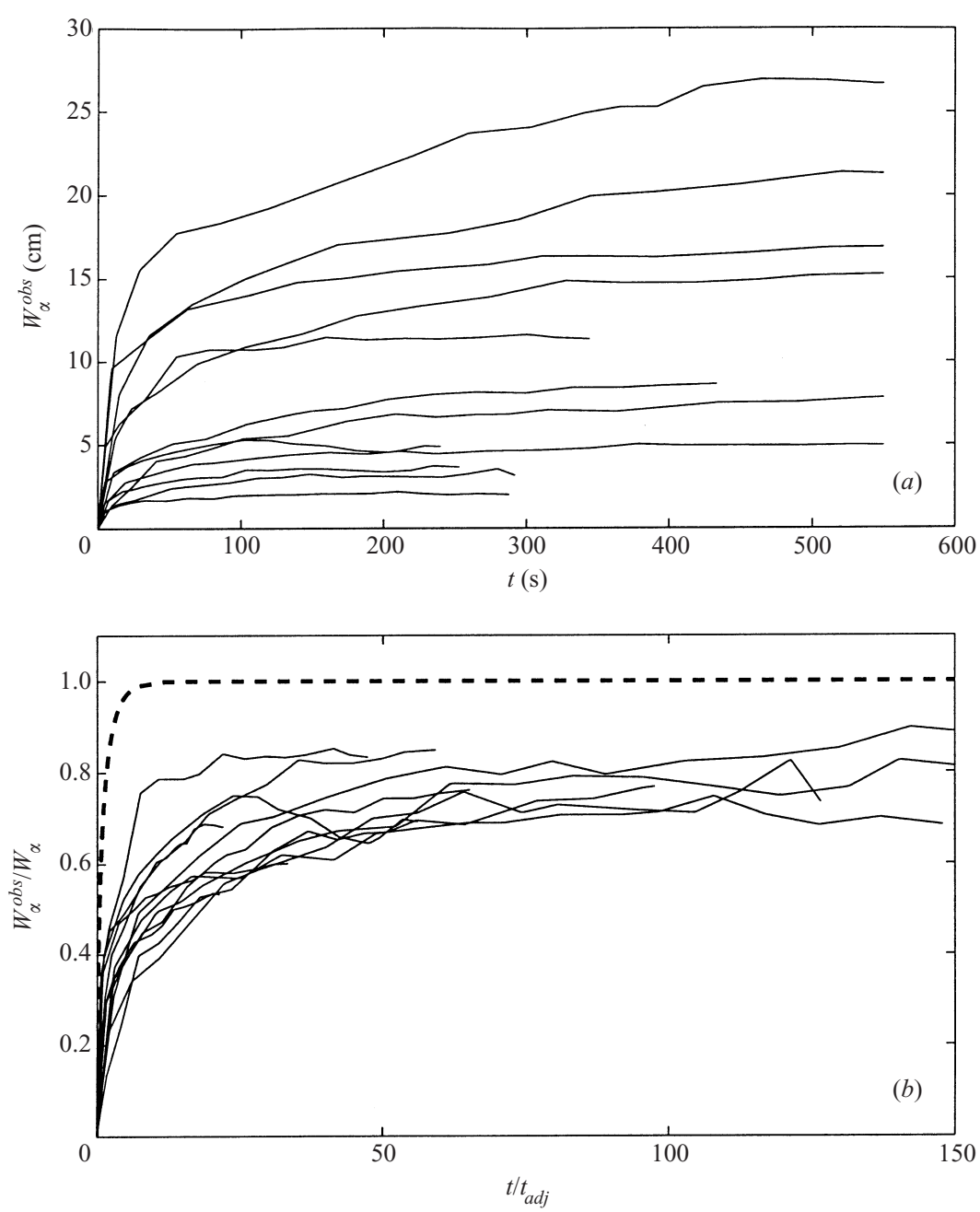

FIGURE 7. (a) The offshore distance to where the front intersects the bottom $W_{\alpha}^{\text {obs }}$ as a function of time for runs LHb. $(b) W_{\alpha}^{o b s}$ normalized by $W_{\alpha}$ as a function of time normalized by $t_{a d j}$. The dashed line in $(b)$ is the theoretical time dependence from (12). The normalized observed widths reach a roughly constant value between $0.7 W_{\alpha}$ and $0.9 W_{\alpha}$.

To quantify the relationship between $W_{\alpha}^{o b s}$ and $W_{\alpha}$, averages of $W_{\alpha}^{o b s}$ over the last five observations of each run were computed for LHa and LHb (figure 8). Including only runs with $c_{w} / c_{\alpha}<7$ that appear to have reached a steady state (these are the runs with $W_{\alpha}<25$ in figure 8), regression analysis yields a slope of $0.67 \pm 0.09$ and a correlation between $W_{\alpha}^{o b s}$ and $W_{\alpha}$ of 0.96 . Including all 28 runs yields a regression slope of $0.54 \pm 0.07$ and a correlation of 0.95 . (Table 3 contains the correlations and regression slopes for this and all subsequent comparisons.) Note that the results are similar for runs LHa (buoyant water dyed) and LHb (ambient water dyed).

\section{Surface width}

In contrast to $W_{\alpha}^{o b s}$, the total gravity current widths $W_{p}^{\text {obs }}$ continue to increase and do not approach a steady width over the duration of each run (figure $9 a$ ). Both $W_{p}^{o b s}$ and the rate of increase vary substantially between runs. For example, $100 \mathrm{~s}$ after the 


\begin{tabular}{ccrccr}
\hline Variable & Data source & Intercept $b$ & Slope $a$ & Correlation & No. runs \\
$W_{\alpha}$ & LHab & $1.25 \pm 1.18$ & $0.68 \pm 0.09$ & 0.96 & 24 \\
$W_{\alpha}$ & YC97 & $0.64 \pm 2.30$ & $0.86 \pm 0.10$ & 0.99 & 9 \\
$W_{\alpha}$ & CL94 & $19.18 \pm 6.11$ & $0.54 \pm 0.19$ & 0.89 & 12 \\
$W_{p}$ & LHa & $0.07 \pm 3.19$ & $1.01 \pm 0.14$ & 0.97 & 16 \\
$W_{p}$ & WCb & $2.24 \pm 1.23$ & $0.35 \pm 0.08$ & 0.92 & 17 \\
$W_{p}$ & G99 & $1.36 \pm 2.11$ & $3.97 \pm 0.27$ & 0.98 & 42 \\
$W_{p}$ & YC97 & $4.58 \pm 4.71$ & $0.92 \pm 0.16$ & 0.98 & 9 \\
$W_{p}$ & CL94 & $30.69 \pm 9.54$ & $0.55 \pm 0.25$ & 0.85 & 12 \\
$c_{p}$ & LHab & $0.15 \pm 0.26$ & $0.86 \pm 0.14$ & 0.93 & 26 \\
$c_{p}$ & WCa* & $-0.05 \pm 0.37$ & $1.08 \pm 0.42$ & 0.89 & 11 \\
$c_{p}$ & WCb & $-0.42 \pm 0.78$ & $1.36 \pm 0.60$ & 0.95 & 6 \\
$u_{p}$ & LHab & $-0.22 \pm 0.85$ & $0.38 \pm 0.22$ & 0.74 & 14 \\
$y_{\max } v s . W_{\alpha}$ & LHab & $5.31 \pm 2.43$ & $0.48 \pm 0.14$ & 0.90 & 14 \\
$y_{\max } v s . W_{\alpha}^{\text {obs }}$ & LHab & $2.02 \pm 2.44$ & $1.04 \pm 0.23$ & 0.94 & 14 \\
& & *excluding run 7 (along wall). & &
\end{tabular}

TABLE 3. Results of regression analyses of the form $x_{o b s}=a x_{s c}+b$ comparing the laboratory and numerical model results $\left(x_{o b s}\right)$ to estimates based on the proposed scaling $\left(x_{s c}\right)$. The \pm values for slope and intercept are $95 \%$ confidence intervals. Data sources are the present laboratory study ( $\mathrm{LHa}$ and $\mathrm{LHb}$ ), the laboratory study of Whitehead \& Chapman (WCa and WCb), the numerical model studies of Garvine (1999) (G99), Chapman \& Lentz (CL94), and Yankovsky \& Chapman (YC97). For $c_{p}$, only runs longer than $2 t_{p}$ are included in the analyses. For $W_{\alpha}$ from LHab only runs that appear to have reached equilibrium $\left(c_{w} / c_{\alpha}<7\right)$ are included in the analyses.

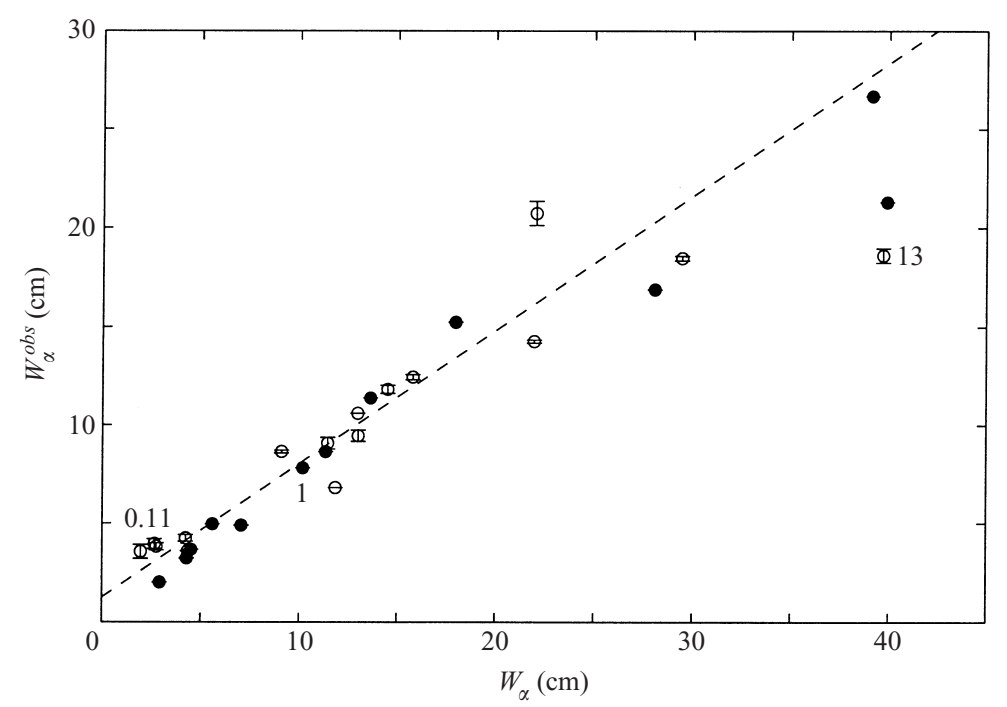

FIGURE 8. Comparison of $W_{\alpha}^{\text {obs }}$ (averaged over the last five samples of each run) and $W_{\alpha}$ for runs LHa (open circles) and LHb (solid circles). For runs that are near equilibrium $\left(c_{w} / c_{\alpha}<7\right)$ the correlation is 0.96 and the regression slope (dashed line) is 0.68 . In this and all subsequent plots the error bars are standard error of the mean (standard deviation divided by the square root of number of samples). The value of $c_{w} / c_{\alpha}$ is noted next to selected points.

nose passes, $W_{p}^{\text {obs }}$ ranges from $20 \mathrm{~cm}$ to $50 \mathrm{~cm}$. Normalizing time by $t_{p}\left(=W_{p} / c_{p}\right)$ and $W_{p}^{o b s}$ by $W_{p}$ collapses all the observations onto a single curve (to the accuracy of the measurements), with $W_{p}^{o b s}$ increasing as roughly $t^{1 / 2}$ (figure $9 b$ ). Using $t_{a d j}$ to normalize time does not collapse the observations. As discussed in $\S 4$, the time 

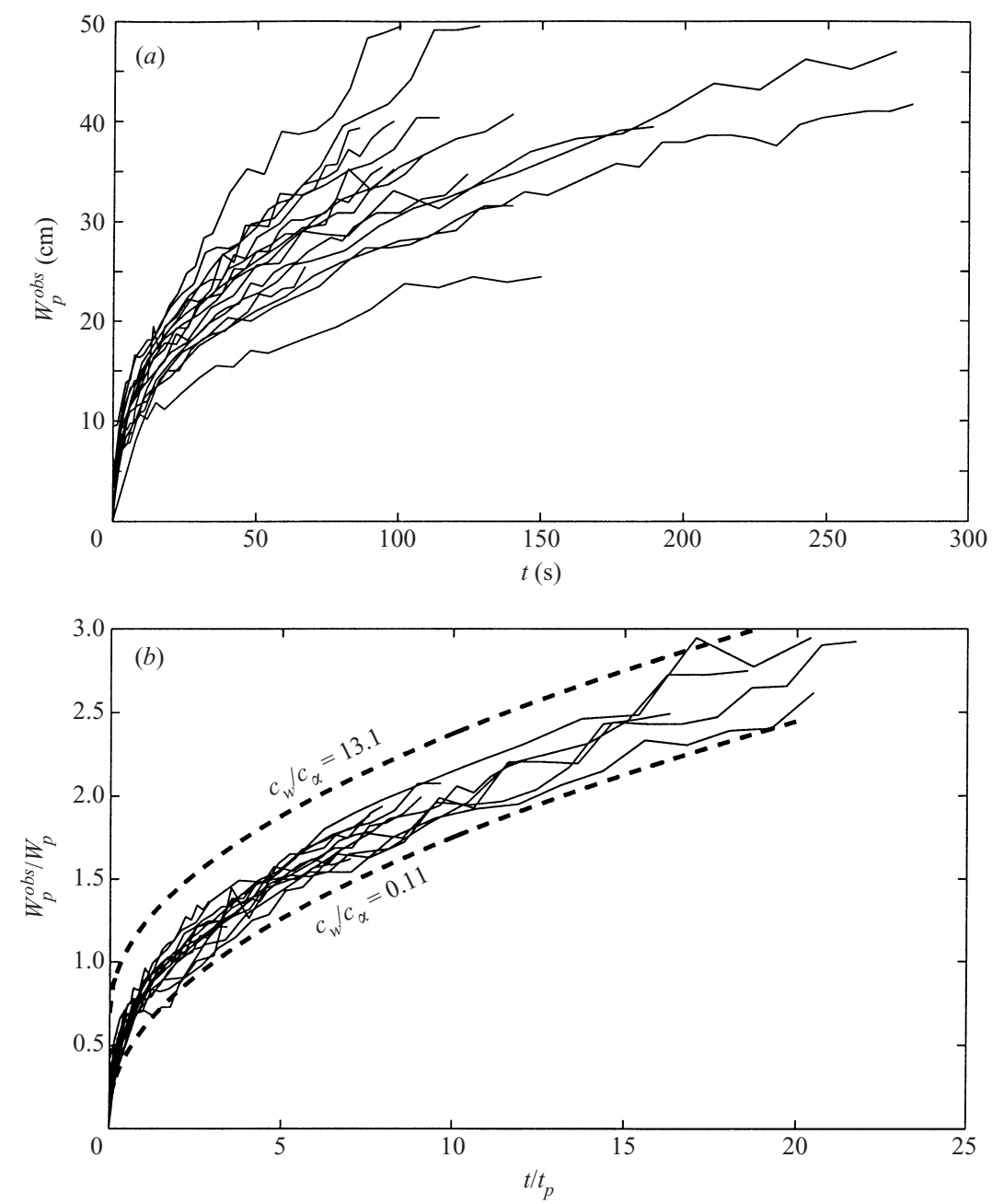

FIGURE 9. (a) The total width $W_{p}^{o b s}$ of the gravity current as a function of time for runs LHa. (b) $W_{p}^{o b s}$ normalized by $W_{p}$ as a function of time normalized by $t_{p}$. The dashed lines in $(b)$ are the estimated time dependence due to interfacial drag from (17) discussed in $\S 4$ for the two experimental limits of $c_{w} / c_{\alpha}$. The time dependence scales as $t^{1 / 2}$.

dependence of $W_{p}^{\text {obs }} / W_{p}$ may be due to interfacial drag between the buoyant plume and the ambient fluid causing the continual increase in gravity current width. The dashed lines are theoretical curves for the time dependence due to the combination of interfacial drag and offshore movement of the foot of the front derived in $\S 4$.

To evaluate the width scaling, $W_{p}^{o b s}$ was averaged over the time period $1<t / t_{p}<3$, and compared to $W_{p}$. The time interval for the averaging was chosen to include all the runs. The shortest run in terms of $t / t_{p}$ was run $4\left(c_{w} / c_{\alpha}=13.1\right)$, which had a duration of $1.4 t_{p}$. The correlation between the average $W_{p}^{\text {obs }}$ over this time interval and $W_{p}$ is 0.97 and the regression slope is $1.01 \pm 0.14$ (figure 10). It is evident from figure $9(b)$ that the correlation is not sensitive to the averaging interval, but the regression slope is sensitive to the choice of averaging interval. The scaling also collapses the gravity current widths reported by Whitehead \& Chapman (1986) from WCb (figure 10 and table 3 ). However, for $\mathrm{WCb}$ the regression coefficient is 0.35 , possibly because the 


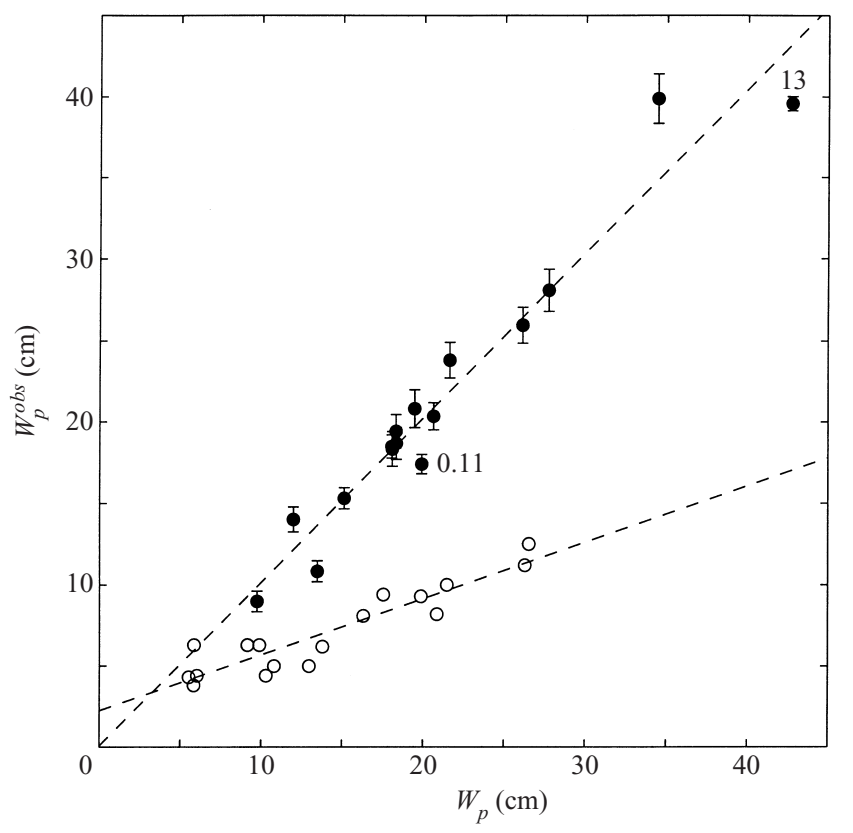

Figure 10. Comparison of $W_{p}^{\text {obs }}$ (averaged over the time interval $1<t / t_{p}<3$ ) and $W_{p}$ for runs LHa (solid circles). The correlation is 0.97 and the regression slope (dashed line) is 1.01 for LHa. Also shown are the results from WCb (open circles) which have a correlation of 0.92 and regression slope (dashed line) of 0.35 . The value of $c_{w} / c_{\alpha}$ is noted next to selected points.

widths were measured shortly after the gravity current encountered the slope, i.e. at an earlier normalized time than in the present laboratory study. As noted previously, their photographs indicate that the gravity current widths continued to increase after the time the width was defined, as also observed in our laboratory runs (figure 9). The buoyancy anomaly $\left(g^{\prime}\right)$ may also be reduced relative to its source value in their runs due to entrainment during the period the gravity current flowed along the wall. Recall that the scaling for $W_{p}(6)$ has two implied coefficients. The comparisons in figure 10 support the choice of 1 for the coefficient multiplying $c_{w} / c_{\alpha}$.

\section{Propagation speed}

Estimates of $c_{p}^{o b s}$ in runs $\mathrm{LHa}$ and $\mathrm{LHb}$ decrease with time from initiation of the source inflow (or equivalently distance from the source) for each run, with the largest variations at the start of the run (figure 11a). Scaling time by $t_{p}$ and $c_{p}^{o b s}$ by $c_{p}$, the large initial variability between runs is primarily confined to $t / t_{p}<2$ (figure $11 b$ ). (In figure $11 b$ the noisy time series of $c_{p}^{o b s}$ were filtered (five-point lowpass filter) to clarify the trends.) At longer times, normalized propagation speeds fall on approximately the same curve, with $c_{p}^{o b s} / c_{p}$ decreasing roughly as $t^{-1 / 2}$. The same time dependence is observed in $\mathrm{WCa}$ and $\mathrm{WCb}$ (not shown, but see figure 7 in Whitehead \& Chapman 1986). The dashed lines again indicate the expected time dependence due to the combination of interfacial drag and offshore movement of the foot of the front $(\S 4)$.

To quantify the relationship between $c_{p}^{o b s}$ and $c_{p}, c_{p}^{o b s}$ (unfiltered data) were averaged over the time period $2<t / t_{p}<4$ (figure 12). (For WCa and WCb the time origin was taken to be when the gravity current began flowing along the slope.) The averaging period was a compromise between obtaining enough of the noisy $c_{p}^{\text {obs }}$ estimates to 

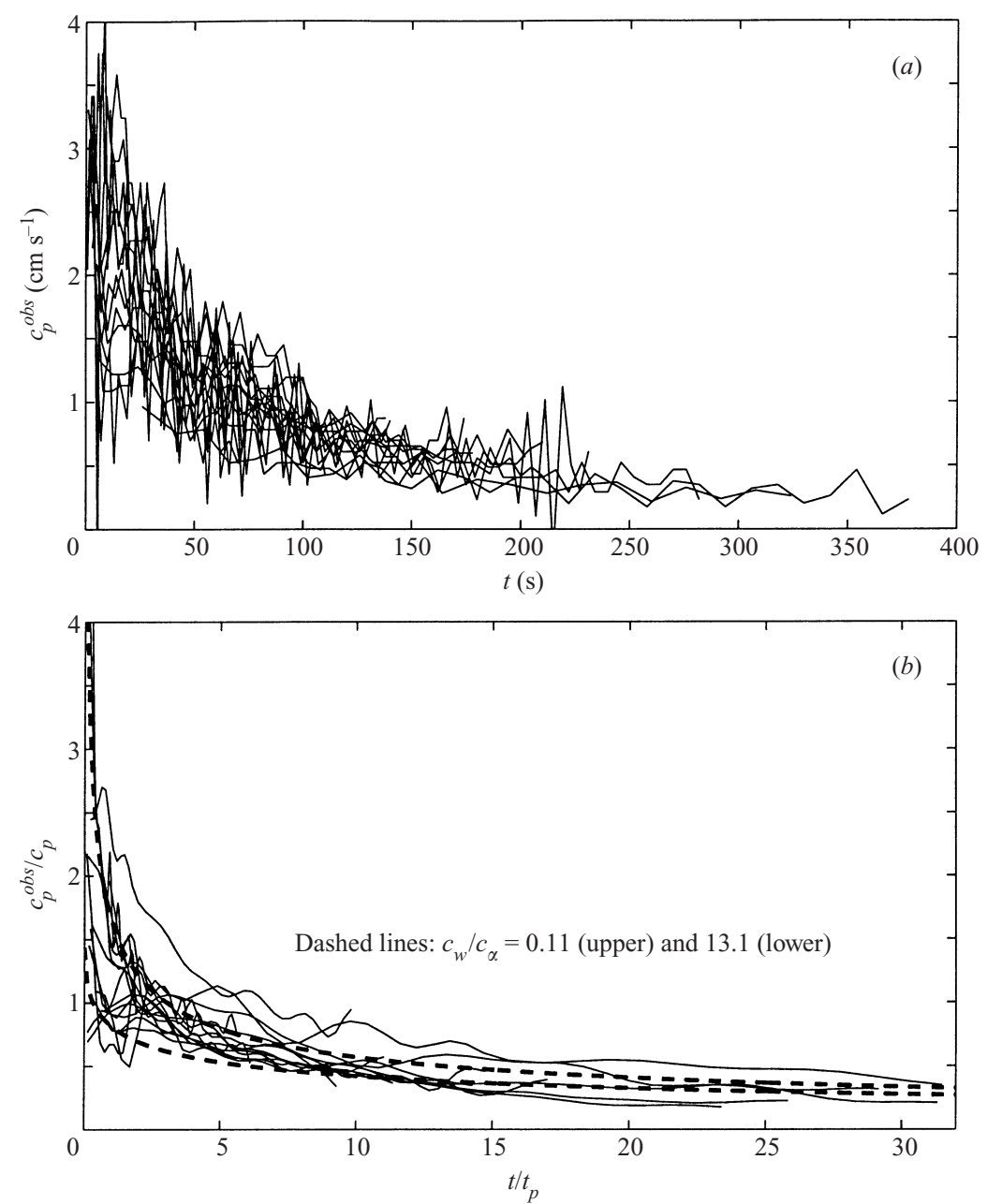

FIGURE 11. (a) The nose propagation speed $c_{p}^{\text {obs }}$ of the gravity current as a function of time for runs LHa. (b) Filtered $c_{p}^{o b s}$ normalized by $c_{p}$ as a function of time normalized by $t_{p}$. The dashed lines in (b) are the estimated time dependence due to interfacial drag from (18) discussed in $\S 4$ for the two experimental limits of $c_{w} / c_{\alpha}$. The time dependence scales as $t^{-1 / 2}$.

form an accurate average and not including too long a period over which $c_{p}^{\text {obs }}$ is decreasing. Two runs from $\mathrm{LHa}$ and $\mathrm{LHb}$ and four runs from $\mathrm{WCb}$ had durations less than $2 t_{p}$; for these runs the averaging interval was $1<t / t_{p}<2$ (open symbols figure 12). Three of the runs have large values of $c_{p}^{o b s}$ relative to $c_{p}$, consistent with the runs not having reached equilibrium. For the $\mathrm{WCa}$ runs the last data point is chosen because the duration was less than $2 t_{p}$ for all but one of these runs. There is close agreement between $c_{p}$ and the average $c_{p}^{o b s}$ for both the present laboratory experiment and the Whitehead \& Chapman study (figure 12, table 3 ). The one outlier from WCa $\left(c_{p}=4.5 \mathrm{~cm} \mathrm{~s}^{-1}\right)$ corresponds to a run along a vertical wall (which was erroneously identified as over a slope in table 1 in Whitehead \& Chapman (1986) (J. Whitehead, personal communication)) and is not included in the regression analysis (table 3). 


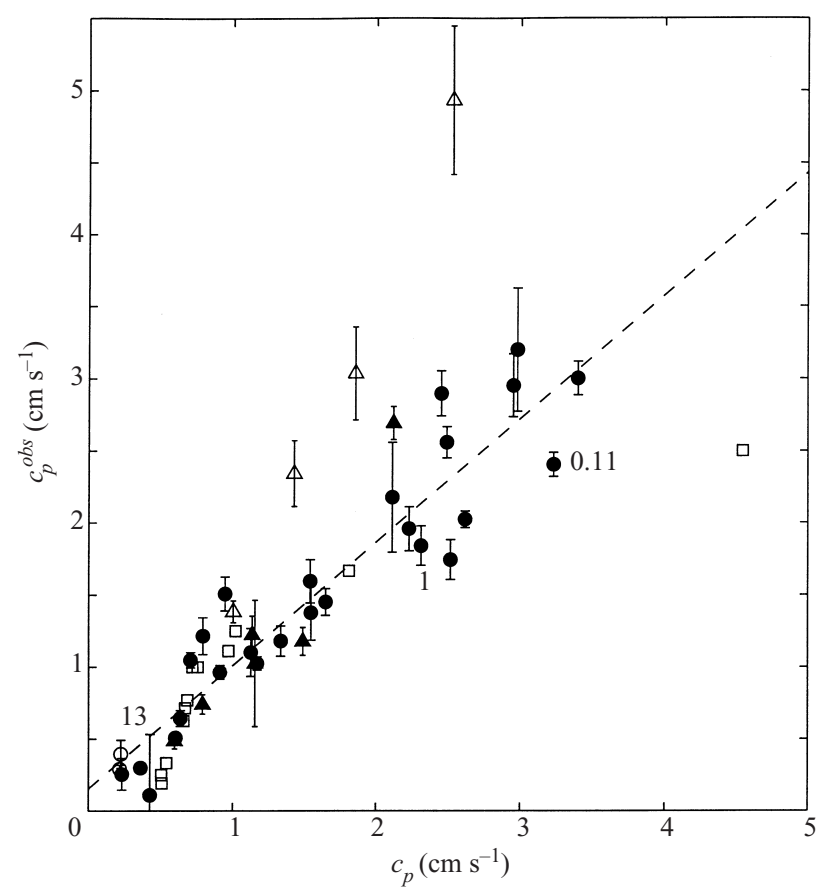

FIGURE 12. Comparison of $c_{p}^{o b s}$ and $c_{p}$. The $c_{p}^{o b s}$ values are averages over the time interval $2<t / t_{p}<4$ (solid symbols) or the time interval $1<t / t_{p}<2$ (open symbols) for runs with durations less than $2 t_{p}$. Runs LHa and LHb are indicated by circles and WCb by triangles. For runs WCa (squares), $c_{p}^{o b s}$ is the last value of each run because of the short durations. The correlation for runs LHa and $\mathrm{L}^{p} \mathrm{Hb}$ is 0.94 and the regression slope (dashed line) is 0.86 (table 3). The correlation and regression slopes are 0.89 and 1.08 for runs $\mathrm{WCa}$ and 0.95 and 1.36 for runs $\mathrm{WCb}$. The value of $c_{w} / c_{\alpha}$ is noted next to selected points.

\section{Surface flow fields}

The observed surface flow field exhibits the qualitative features inferred from the scaling theory (see figure 3). An example of the flow field for a surface-trapped gravity current $\left(c_{w} / c_{\alpha}=0.17\right.$, run 16$)$, is shown in figure 13. The along-slope current is largest near the coast $\left(W_{\alpha}=2.7 \mathrm{~cm}\right)$ and decreases with distance offshore (figure $\left.13 a, b\right)$. There is also an offshore flow that is not consistent with the scaling, but is consistent with the gravity current width increasing with time (figure 9 and $\S 4$ ). Near the nose, the flow moves parallel to the coast at about the nose speed. In a coordinate frame moving with the nose, the flow is toward the nose near the coast, turns and is away from the nose farther offshore (figure $13 c$ ). Note the resemblance to the flow field for a gravity current along a wall (figure 1). For a slope-controlled gravity current (run $7, c_{w} / c_{\alpha}=3.8$ in figure $14 a, b$ ), the along-slope current is weak near the coast and increases with distance offshore, reaching a maximum near $W_{\alpha}(=22 \mathrm{~cm})$. In a coordinate frame moving with the nose, the flow is toward the nose over the outer part of the gravity current, turns onshore near the nose, and is away from the nose near the coast (figure 14c). Thus, in a coordinate frame moving with the nose, the two limits of $c_{w} / c_{\alpha}$ exhibit opposite flow patterns.

To evaluate the proposed velocity scale $u_{p}$, (9), along-slope velocities as a function of offshore distance were determined for all the surface drifters in a given run between 40 and $160 \mathrm{~cm}$ downstream of the source (figure 15). The along-slope velocities were averaged over $2 \mathrm{~cm}$ intervals extending offshore to form average cross-slope profiles. 

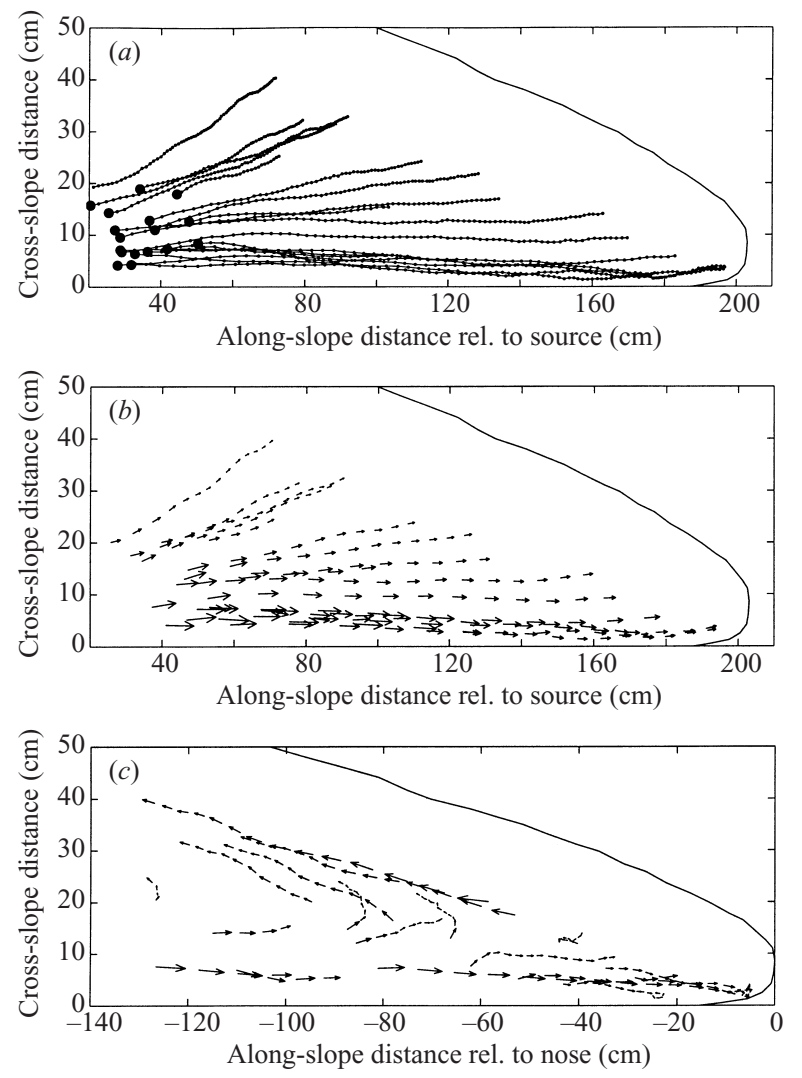

FIGURE 13. The flow field from run $16, c_{w} / c_{\alpha}=0.17,(a)$ individual particle tracks, $(b)$ velocity vectors in the stationary reference frame, and $(c)$ velocity vectors in a reference frame moving with the nose of the gravity current. The outline of the plume near the end of the run is also shown for reference in each panel.

The resulting profiles may not be representative of equilibrium conditions because the averages necessarily include periods prior to the foot of the front reaching its equilibrium position and span a broad period of increasing gravity current width and decreasing propagation speed. In each profile there is a maximum in the along-slope velocity, $u_{\max }$, that is near $W_{\alpha}^{o b s}$ and hence is farther offshore as $c_{w} / c_{\alpha}$ increases. In general, the velocity onshore of $W_{\alpha}^{\text {obs }}$ is small, but not zero as assumed (figure 15), possibly because the gravity currents have not reached equilibrium or the front may not be sharp due to mixing. To quantify these relationships, the maximum of the average along-slope velocity profile $u_{\max }$ and the offshore position of the maximum $y_{\max }$ were determined for each run. In figure $16(a) u_{\max }$ is plotted against $u_{p}=c_{w}$. The correlation is 0.74 and the regression slope is 0.38. In figure $16(b) y_{\max }$ is plotted versus $W_{\alpha}$ (and $W_{\alpha}^{o b s}$ ). Except for two runs with large $c_{w} / c_{\alpha}(=5.8$ and 13$), y_{\max }$ is approximately equal to $W_{\alpha}$. The two outliers are almost certainly because these gravity currents with large $c_{w} / c_{\alpha}$ have not reached equilibrium (i.e. $W_{\alpha}^{\text {obs }}<W_{\alpha}$ ), as discussed above. Comparison of $y_{\max }$ with the observed position of the foot of the front $W_{\alpha}^{o b s}$ (open circles figure $16 b$ ) instead of $W_{\alpha}$, indicates $y_{\max }$ is near $W_{\alpha}^{\text {obs }}$. The regression slope between $W_{\alpha}^{o b s}$ and $y_{\max }$ is about 1.0 and the correlation is 0.94 (table 3). 

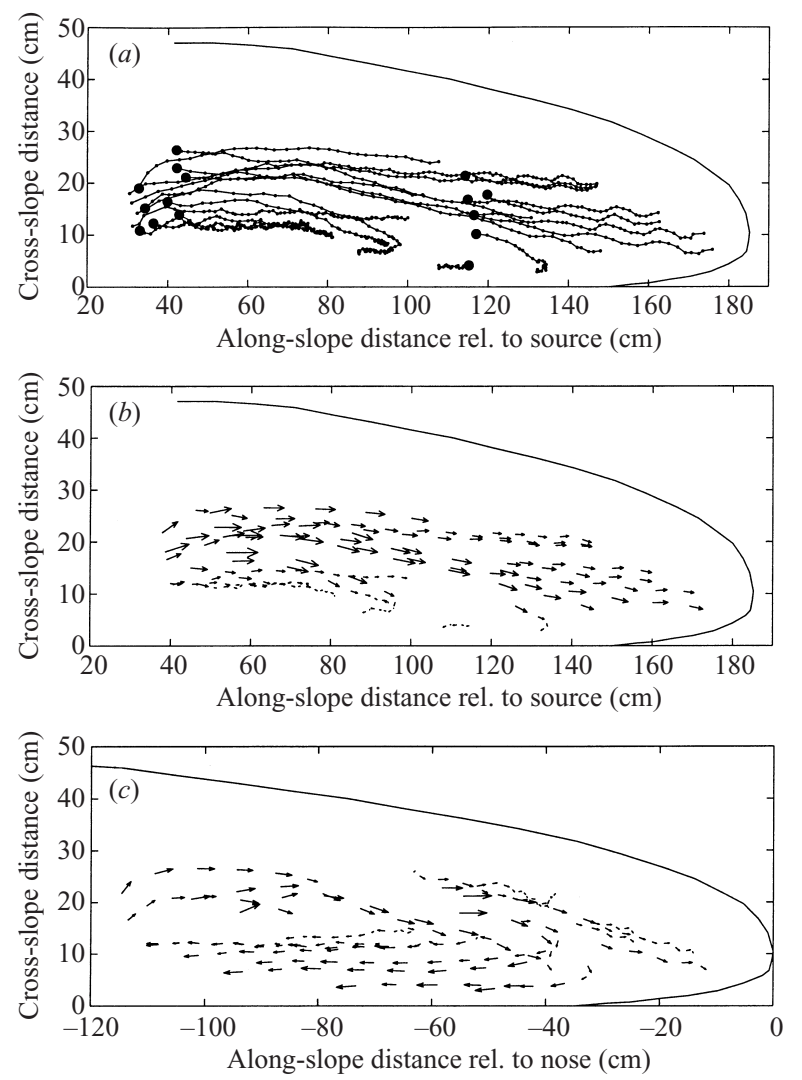

FIGURE 14 . The flow field from run $7, c_{w} / c_{\alpha}=3.8,(a)$ individual particle tracks, $(b)$ velocity vectors in the stationary reference frame, and $(c)$ velocity vectors in a reference frame moving with the nose of the gravity current. The outline of the plume near the end of the run is also shown for reference in each panel.

\subsection{Evaluation of the scaling using numerical model results}

Three numerical modelling studies of buoyant water discharge onto a continental shelf provide independent tests of the proposed scaling. Since the objective of these studies is to understand coastal gravity currents the models have geometries that are more consistent with geophysical applications, e.g. small bottom slopes. Garvine (1999) used a numerical model to investigate how far along the coast a gravity current would extend before it was completely dispersed by mixing with the ambient shelf water. Chapman \& Lentz (1994) and Yankovsky \& Chapman (1997) used numerical models to study the dynamics of slope-controlled gravity currents. The numerical models used in these three studies are hydrostatic, primitive-equation, regional circulation models. Details of the numerical model configurations can be found in the cited articles. These three studies were chosen because they each included a sloping bottom, a large number of model runs spanning a broad range of parameter space, and gravity current widths are reported that can be compared to the scale width $W_{p}$. Chapman \& Lentz (1994) and Yankovsky \& Chapman (1997) also report offshore distances to the foot of the front that can be compared to $W_{\alpha}$. None of these studies reported information on the nose propagation speed $c_{p}$. Garvine's study included 66 model runs in which $c_{w} / c_{\alpha}$ ranged from 0 to 2 , with $80 \%$ of the runs having values less 

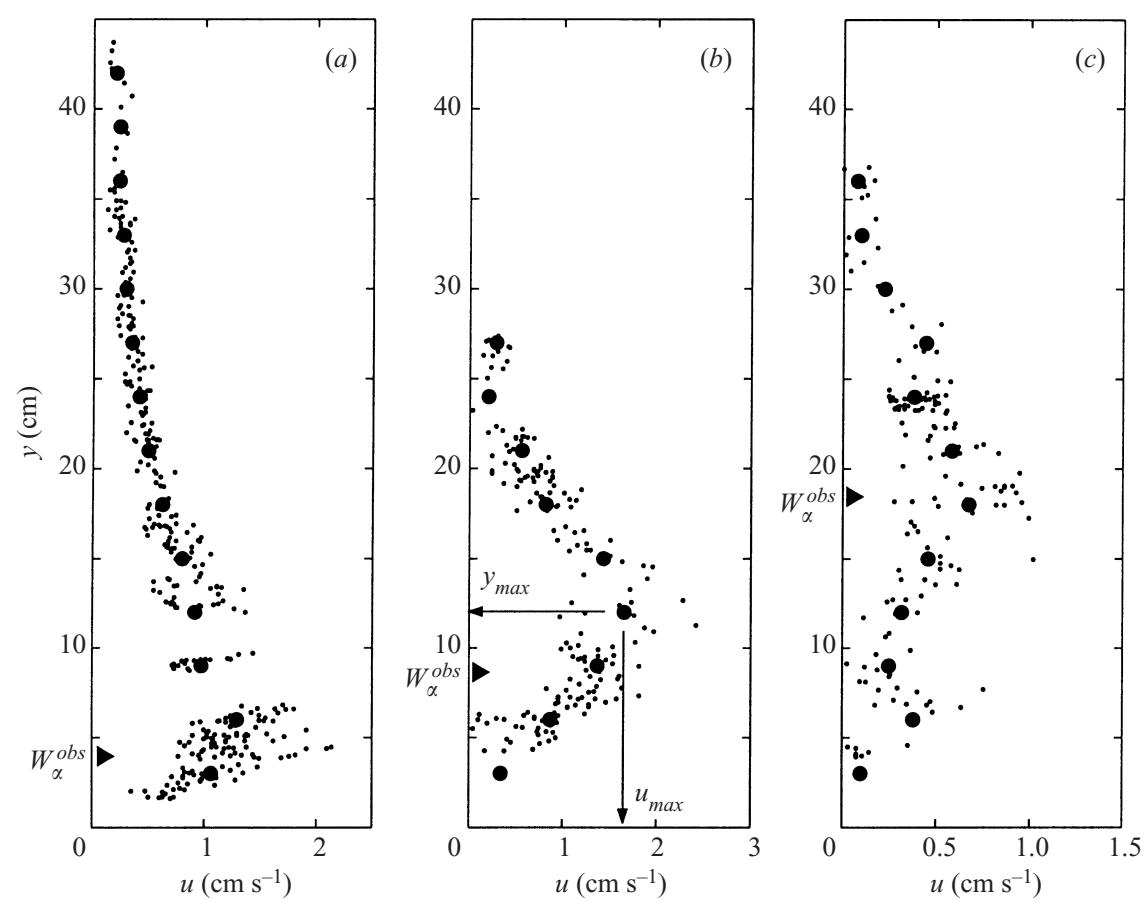

FIGURE 15. Cross-slope profiles of along-slope velocity $u$ for $(a) c_{w} / c_{\alpha}=0.17$ (run 16), (b) $c_{w} / c_{\alpha}=1.01$ (run 6), and (c) $c_{w} / c_{\alpha}=5.91$ (run 10). Small dots represent individual velocity estimates from the difference in particle positions between frames. Large circles represent average velocity in $2 \mathrm{~cm}$ cross-slope bins. The maximum along-slope velocity $u_{\max }$ and the cross-slope location $y_{\max }$ for the bin-averaged profiles are determined for each run. The locations of $W_{\alpha}^{\text {obs }}$ are indicated by the triangles.

than 1. The numerical model runs by Chapman \& Lentz (1994) (12 model runs) and Yankovsky \& Chapman (1997) (nine model runs) focused on slope-controlled gravity currents for which $c_{w} / c_{\alpha}$ ranged from 1 to 13 .

Garvine (1999) varied $Q, \Delta \rho, \alpha$, source widths and latitude $(f)$. The gravity currents only penetrate a finite distance along the coast because of mixing. Garvine estimated the width of the gravity current at a position that was about halfway between the source and the total along-coast penetration distance of the gravity current. Garvine then determined empirically the dependence of gravity current width on various input parameters for those runs with a constant bottom slope of 0.002 (summarized in his figure 13). To determine whether $W_{p}$ collapses Garvine's numerical model results, estimates of $W_{p}$ from (6) are compared to the model buoyancy current widths $W_{p}^{\text {model }}$ (figure 17). The model widths are proportional to $W_{p}$ for the model runs in which $Q, \Delta \rho, \alpha$ and inflow width were varied, but not for the model runs at small $f$ (latitudes less than $25^{\circ}$ ). Excluding the low latitude runs, the correlation is 0.98 (table 3). Moreover, (6) accounts for the dependence on bottom slope that was not included in Garvine's empirical relationship. The regression coefficient of about 4 between $W_{p}^{\text {model }}$ and $W_{p}$ presumably accounts for the relationship between the source and local gravity current values of $Q$ and $g^{\prime}$. There is a substantial reduction in $g^{\prime}$ between the source and where the width was measured due to mixing with the ambient fluid. The failure of the scaling at low latitudes is probably because the relationship between the inlet transport and the coastal gravity current transport is 

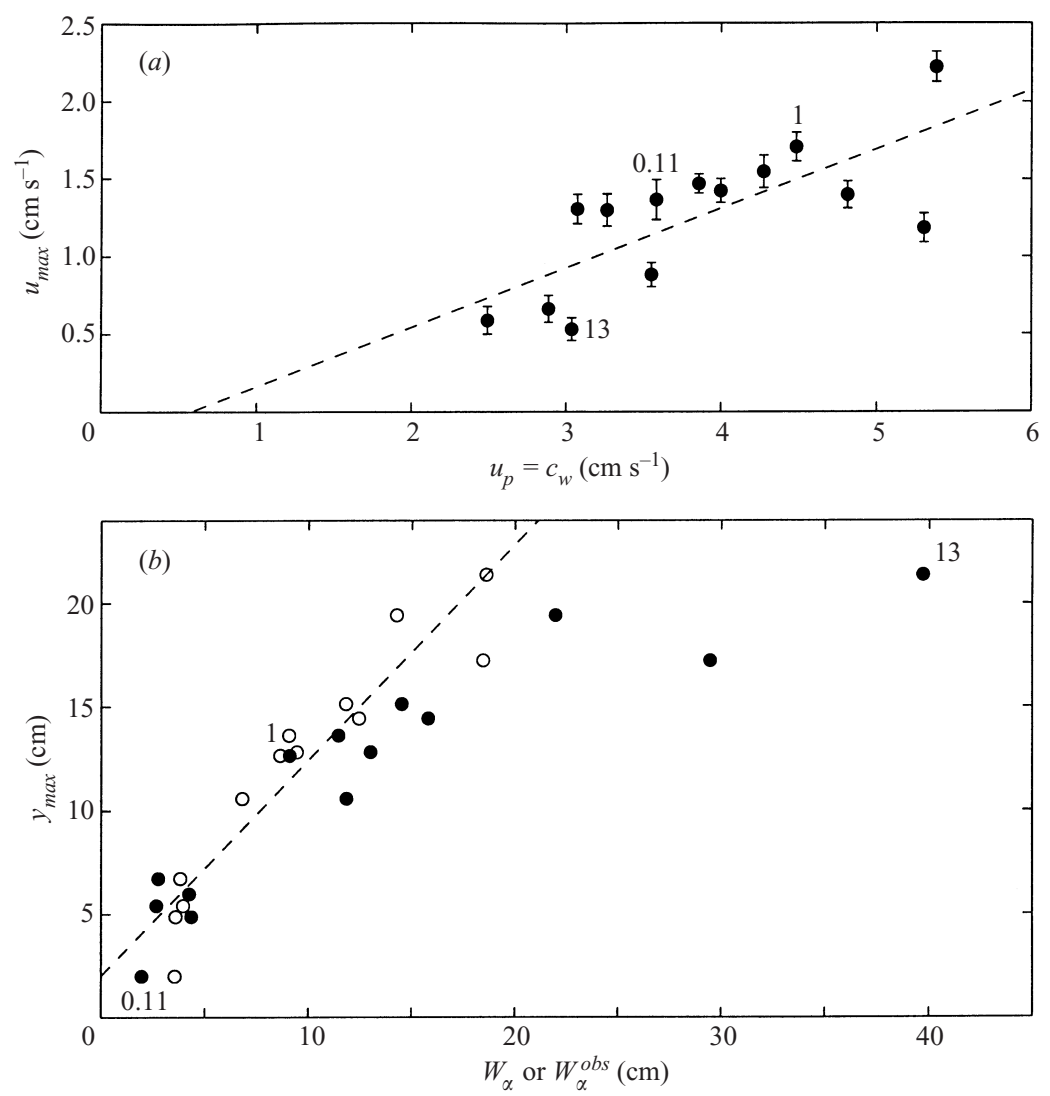

FIGURE 16. (a) Comparison of maximum along-slope flow from the mean cross-slope profile $u_{\max }$ and $u_{p}$ for runs LHa. The correlation is 0.74 and the regression slope (dashed line) is 0.38 . (b) Comparisons of cross-slope position of current maximum $y_{\max }$ with $W_{\alpha}$ (solid circles) and with $W_{\alpha}^{o b s}$ (open circles) for runs LHa. The correlation between $y_{\max }$ and $W_{\alpha}^{\text {obs }}$ is 0.94 and the regression slope (dashed line) is 1.04 . The value of $c_{w} / c_{\alpha}$ is noted next to selected points.

a strong function of $f$ (Garvine 2001). As $f$ decreases, an increasing fraction of the source transport goes into a growing recirculation cell near the source, until for zero rotation there is no coastal gravity current (see figures 7 and 8 in Garvine 1999). Since the coastal current transports where the width was measured were not reported, it was not possible to check whether the scaling would collapse the low-latitude model results if the local transport were used.

Chapman \& Lentz (1994) and Yankovsky \& Chapman (1997) conducted numerical model runs in which $Q, \Delta \rho$ and $\alpha$ were varied. Results from two of the model runs from Chapman \& Lentz (1994) are not considered here because the buoyant gravity current had clearly not reached steady state, i.e. it was still spreading offshore. In the Chapman \& Lentz (1994) model runs, a portion of the buoyant water spreads upstream of the source (the opposite direction to the gravity current propagation) indicating that the transport in the buoyant gravity current is somewhat smaller than the source transport. Chapman \& Lentz (1994) found that this upstream spreading was eliminated by imposing a weak ambient current in the direction of the gravity current propagation. Therefore, Yankovsky \& Chapman (1997) imposed a weak ambient current $\left(4 \mathrm{~cm} \mathrm{~s}^{-1}\right)$ in all of their model runs to ensure that the transport 


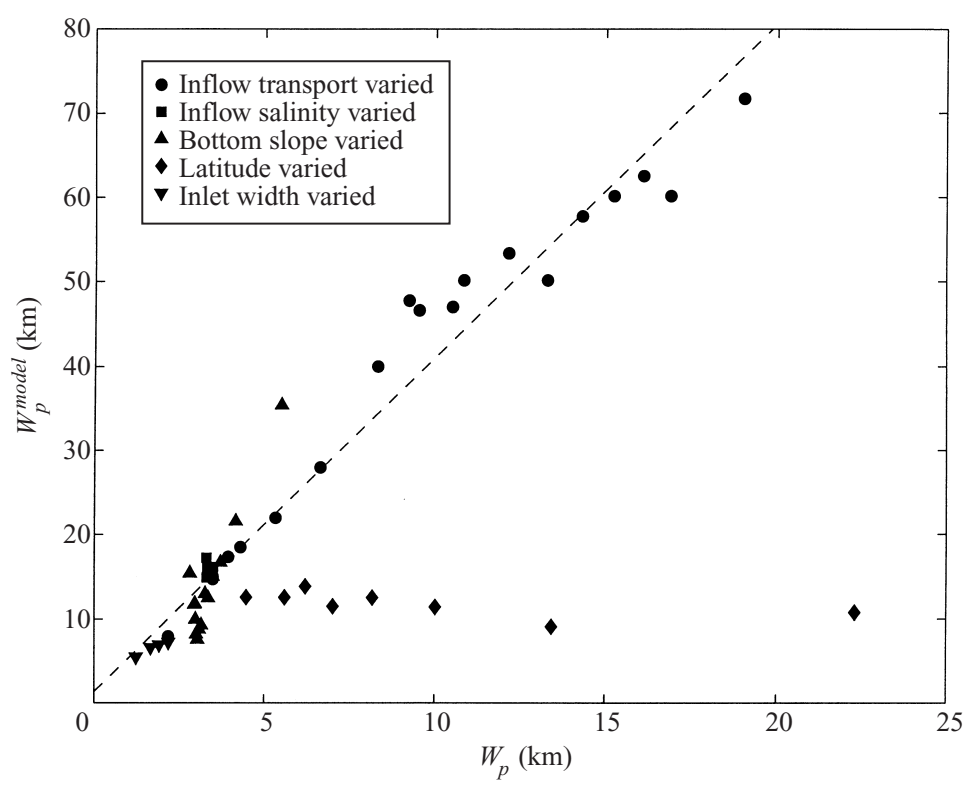

FIGURE 17. Comparison of gravity current width $W_{p}^{\text {model }}$ with $W_{p}$ from Garvine's (1999) numerical model runs. The various run types are indicated in the inset. Excluding runs with latitudes $(f)$ less than $25^{\circ}$, the correlation is 0.98 and the regression slope (dashed line) is 3.97.

in the buoyant gravity current equalled the source transport. The resulting gravity current widths from their model studies, $W_{p}^{\text {model }}$, are proportional to $W_{p}$ (figure 18 ) with regression slopes of 0.55 and 0.92 (table 3). The smaller regression slope for the Chapman \& Lentz (1994) results may be related to the upstream spreading of some of the buoyant water from the source. Both studies also reported the offshore distances to the foot of the front $W_{\alpha}^{\text {model }}$, which agree well with $W_{\alpha}$ (table 3), as previously reported by Yankovsky \& Chapman (1997). There is relatively little change in the density of the buoyant gravity current in the numerical model runs of both Chapman \& Lentz (1994) and Yankovsky \& Chapman (1997) compared to the model runs of Garvine (1999), indicating much less mixing with the ambient shelf water. Therefore, the local $Q$ and $g^{\prime}$ of the buoyant current are closer to the inflow (source) values.

\section{Discussion}

The laboratory experiments and the reanalysed numerical model results support the proposed scaling in almost every measure over two orders of magnitude in the parameter $c_{w} / c_{\alpha}$. The demonstration that the front adjusts to a steady offshore position that scales as $W_{\alpha}$ (figures 7 and 8), is the first laboratory confirmation of the slope-controlled gravity current equilibrium (Chapman \& Lentz 1994; Yankovsky \& Chapman 1997).

However, one aspect of the laboratory observations that is not consistent with the scaling theory is the temporal increase in $W_{p}^{o b s}$ and corresponding decrease in $c_{p}^{o b s}$. In the absence of entrainment, the assumption was made that the gravity current would reach an equilibrium and then the geometry and propagation speed would not change. The fact that $W_{p}^{o b s}$ increases as $t^{1 / 2}$ (figure $9 b$ ) and $c_{p}^{o b s}$ decreases as $t^{-1 / 2}$ (figure $11 b$ ) is consistent with conserving volume transport and $h_{p}$, or equivalently $W_{\alpha}^{o b s}$, reaching a steady value (figure 7). Stern et al. (1982) and Griffiths \& Hopfinger 


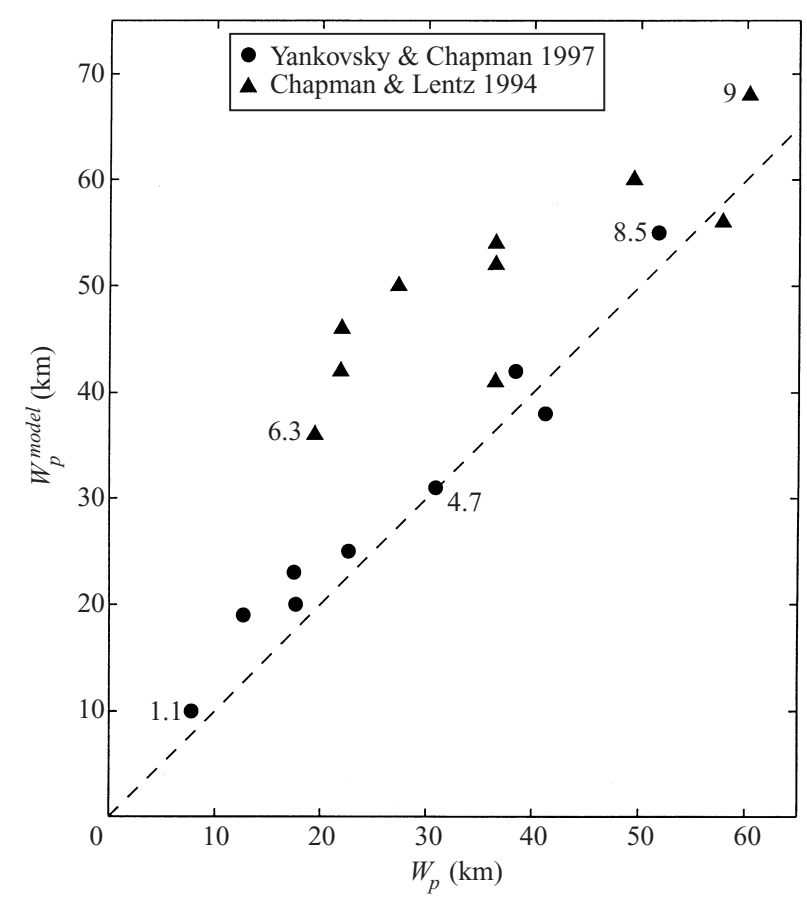

Figure 18. Comparison of $W_{p}^{\text {model }}$ with $W_{p}$ from Chapman \& Lentz's (1994) and Yankovsky \& Chapman's (1997) numerical model runs. Values of $c_{w} / c_{\alpha}$ are noted for selected numerical model runs.

(1983) observed a weak exponential decay of the nose speed of gravity currents along a wall that Griffiths \& Hopfinger (1983) attributed to radiation of inertial waves. Griffiths (1986) suggests that continual detrainment of buoyant plume fluid from the nose region also may lead to a slow decay of speed. The finite size of the upstream reservoirs also may lead to an eventual reduction in nose speed as the initially upstream-propagating Kelvin wave generated by the dam break is reflected downstream and eventually catches up with the nose (cf. Rottman \& Simpson 1983). Kubokawa's (1984b) experiments along a wall showed little evidence of nose speed decay, perhaps because of the relatively large upstream basin.

The nose propagation speed along the slope in Whitehead \& Chapman's experiments also exhibited a $t^{-1 / 2}$ dependence that they attributed to downstream radiation of topographic shelf waves. Interestingly, the increasing width is not evident in numerical model runs (Chapman \& Lentz 1994; Yankovsky \& Chapman 1997) and the decreasing propagation speed with time is not evident in limited oceanic observations (Rennie et al. 1997). This suggests that the temporal evolution of $c_{p}$ and $W_{p}$ may be associated with a process specific to the laboratory. One possible explanation for the $t^{-1 / 2}$ dependence of $c_{p}$ is continuous entrainment of ambient fluid into the buoyant current that reduces $g^{\prime}$ (Rennie et al. 1997). However, there was little evidence of significant entrainment in the new laboratory runs from either the video images or the measurements of downstream plume density. Furthermore, the agreement between the time dependence of $W_{\alpha}^{o b s}$ and the theoretical time dependence (2) that assumes no change in $g^{\prime}$ (figure 7) suggests entrainment is not the cause of the time dependence.

An alternative explanation is that viscous, interfacial drag between the buoyant 
current and ambient fluid at rest is widening and slowing the gravity current. The resulting stress-driven Ekman flow would move the front offshore near the surface (increase $W_{p}$, but not affect $W_{\alpha}$ ) and retard the along-slope flow. From Ekman layer dynamics, if the geostrophic flow in the gravity current is $u_{p}$, the stress-driven component of flow $\left(u_{E}, v_{E}\right) \sim\left(-u_{p} / 4, u_{p} / 4\right)$, oriented at $45^{\circ}$ to the along-slope direction, and it occurs in an Ekman layer of thickness $(2 v / f)^{1 / 2}=1.5-3 \mathrm{~mm}$, where $v$ is the kinematic viscosity of water. This is qualitatively consistent with the observed flow pattern in figure 13. To test this hypothesis, consider first the influence of this stressdriven flow on the width of the gravity current offshore of the foot of the front $W_{s}(t)$ (to distinguish this time-dependent width from the width scale $W_{w}$ ). Assume a fixed transport $Q$ confined offshore of the foot of the front, as before, so

$$
Q \sim u \frac{W_{s} h_{p}}{2},
$$

where $u$ is the along-slope geostrophic velocity in the frontal region. Assume $h_{p}$ is fixed, but that the width $W_{s}$ increases at a rate proportional to the offshore Ekman drift

$$
\frac{\partial W_{s}}{\partial t} \sim v_{E}=\frac{u}{4} .
$$

Using (15) to substitute for $u$ in (14), noting that $Q / h_{p} \sim f W_{w}^{2} / 2$ (see (9)) and integrating in time yields

$$
W_{s} \sim W_{w}\left(\frac{t f}{2}\right)^{1 / 2},
$$

where we assume that $W_{s}=0$ at $t=0$. The total width $W(t) \sim W_{s}(t)+W_{\alpha}$ normalized by $W_{p} \sim W_{w}\left(1+c_{w} / c_{\alpha}\right)$ is

$$
\frac{W}{W_{p}} \sim\left(\frac{t}{2 t_{p}}\right)^{1 / 2}+\frac{c_{w} / c_{\alpha}}{1+c_{w} / c_{\alpha}}
$$

where $t_{p}=W_{p} / c_{p}=\left(1+c_{w} / c_{\alpha}\right)^{2} / f$. For simplicity, we focus on times long compared to the rapid adjustment of the foot of the front to its offshore equilibrium position. Therefore we neglect the time dependence of $h$ given by (12) and assume the depth of the foot of the front is constant and equal to $h_{p}$. The time dependence associated with the adjustment of the foot of the front can be incorporated into this analysis by integrating numerically, but the changes are small and do not alter the basic $t^{1 / 2}$ behaviour. The time dependence of the nose propagation speed $c(t)$ can be estimated by assuming $c(t) \sim Q / A(t)$ as in (7), where, in this case, $A(t) \sim W(t) h_{p} / 2$. Using (17) and (12) yields

$$
\frac{c}{c_{p}} \sim\left[\left(\frac{t}{2 t_{p}}\right)^{1 / 2}+\frac{c_{w} / c_{\alpha}}{1+c_{w} / c_{\alpha}}\right]^{-1} .
$$

The estimated time dependence due to interfacial drag, $W(t) / W_{p}$ from (17) and $c(t) / c_{p}$ from (18) are shown in figures 9 and 11 (dashed lines) for the minimum and maximum values of $c_{w} / c_{\alpha}$. A proportionality constant of 0.75 for $W$, and the inverse for $c$ have been used to roughly fit the data. In both cases the essential feature is the $t^{1 / 2}$ behaviour of $W_{s}$ in (16) and $t^{-1 / 2}$ dependence of $c$ in (18). The agreement supports the hypothesis that the continual increase in the gravity current widths observed in both our experiments and those of Whitehead \& Chapman (1986) 
are due to interfacial viscous drag between the buoyant plume and ambient fluids. While this process is important in the laboratory studies, it may not be important in the ocean (or numerical models with stratification-dependent mixing schemes) where viscous drag is small and turbulent stresses at the interface may be inhibited by the strong stratification separating the gravity current and the ambient fluid.

For our laboratory experiments, the constants associated with the width $W_{p}$ and nose propagation speed $c_{p}$ are approximately 1 based on either the regression analyses (table 3) or the coefficients used for the derived time dependence (figures 9 and 11). This is consistent with the corresponding constants found in the previous laboratory studies of gravity currents along a wall (Stern et al. 1982; Griffiths \& Hopfinger 1983; Kubokawa \& Hanawa 1984b). One of the striking contrasts to previous studies of gravity currents along a wall is the stability of the flow in the presence of a slope (figure 5). Over a sloping bottom there was little evidence of mixing in the nose region. Griffiths \& Hopfinger (1983) concluded that mixing near the nose was due to Kelvin-Helmholtz instability for gravity currents against a vertical wall. Neither was there indication of baroclinic instability in the trailing geostrophic current over a sloping bottom, in contrast to the vertical wall studies (Griffiths \& Linden 1981, 1982; Griffiths \& Hopfinger 1983). The observed stability is consistent with linear stability analysis of coastal upwelling fronts over a sloping bottom (Barth 1989a, b; Reszka \& Swaters 1999). The elimination of the Kelvin-Helmholtz billows is not due solely to Reynolds number effects. In our experiments an estimate of the Reynolds number in the nose region is $R e=\left(u_{p} h_{p}\right) / v \approx 800-2000$, comparable to those of Stern et al. (1982) and Griffiths \& Hopfinger (1883). One possible explanation is that the shallow layer under the nose region prevents billows from growing to large amplitude. Another possibility is that the vertical shear is reduced by the presence of the slope. Recall that some experiments were conducted for $c_{w} / c_{\alpha}<0.2$, well into the wall regime, and that the nose speeds and current widths approached the earlier results for gravity currents along a vertical wall. However, even for these low values of $c_{w} / c_{\alpha}$ the slope was at most $\alpha=0.29$. It remains to be seen if nose region turbulence and baroclinic instabilities occur for larger, but finite, values of $\alpha$, or whether the vertical wall is a special case.

It is premature to claim that the scaling coefficients that have been determined from the experiments are valid at oceanographic scales. However, it is useful to ask where parameters typical of the coastal ocean lie in the scaling. Continental shelves generally have bottom slopes in the range $\alpha=10^{-4}$ to $10^{-2}$. For a typical mid-latitude estuary with a moderate outflow $Q=500 \mathrm{~m}^{3} \mathrm{~s}^{-1}, f=10^{-4} \mathrm{~s}^{-1}$, and $g^{\prime}=0.02 \mathrm{~m} \mathrm{~s}^{-2}$ (representative of Delaware Bay; Garvine 1999). For these values, with $\alpha=10^{-4}$ to $10^{-2}$, (8) gives $10>c_{w} / c_{\alpha}>0.1$. The weak $Q^{1 / 4}$ dependence of $c_{w} / c_{\alpha}$ makes this estimate relatively insensitive to the source transport, i.e. increasing the transport by a factor of 100 (representative of a river such as the Mississippi) only increases $c_{w} / c_{\alpha}$ by a factor of 3 . Thus, oceanic buoyant coastal currents are often in the intermediate range between the surface-trapped and slope-controlled limits. The vertical wall limit, while providing insight, is probably not directly applicable to most oceanographic situations. One consequence is that in oceanographic settings, the flow field may be quite different from that observed in the classical laboratory studies of gravity currents along a vertical wall, with the along-slope flow concentrated offshore near the front and onshore flow near the nose (figure 14). This flow pattern and the rate at which fluid moves along the shelf may have implications for the coastal ocean since buoyant coastal currents transport constituents, such as sediment, marine organisms, nutrients and chemical pollutants. 


\section{Summary}

A simple scaling theory is proposed for buoyant gravity currents over a sloping bottom that extends and builds on previous studies of gravity currents along a wall (Stern et al. 1982; Griffiths \& Hopfinger 1983; Kubokawa \& Hanawa, 1984b) and gravity currents in contact with a sloping bottom (Chapman \& Lentz 1994; Yankovsky \& Chapman 1997). The proposed theory provides scales for the buoyant current thickness $h_{p}(1)$, the width $W_{p}(6)$, nose propagation speed $c_{p}(7)$, and the flow field characteristics $((9)$, and figure 3$)$ given the gravity current transport $Q$, the density anomaly $g^{\prime}\left(=g \Delta \rho / \rho_{\circ}\right)$, the Coriolis frequency $f$ and the bottom slope $\alpha$. Key assumptions are: the cross-slope momentum balance is geostrophic; the foot of the front moves offshore until the near-bottom flow, and hence the bottom stress, are zero; and the gravity current nose shape remains roughly steady as it propagates along the coast. The key non-dimensional parameter characterizing the buoyant gravity current response is $c_{w} / c_{\alpha}$, where $c_{w}=\left(2 Q g^{\prime} f\right)^{1 / 4}$ is the nose speed limit over a steep bottom slope (vertical wall) and $c_{\alpha}=\alpha g^{\prime} / f$ is the nose speed limit over a gently sloping bottom. If $c_{w} / c_{\alpha} \ll 1$, then the gravity current is surface-trapped, independent of the bottom slope and resembles a gravity current along a vertical wall. In this case the gravity current width is approximately the deformation radius based on the gravity current thickness $h_{p}$ and both the nose speed and the average flow speed are approximately $c_{w}$. If $c_{w} / c_{\alpha} \gg 1$, then the gravity current is slope-controlled, i.e. most of the buoyant water is in contact with the bottom and bottom friction is important in the establishment of the current. In this case the gravity current width is $1+c_{w} / c_{\alpha}$ times the deformation radius based on $h_{p}$ and the nose propagation speed is approximately $c_{\alpha}$. Behind the nose, the along-slope flow is concentrated at the front separating the buoyant and ambient fluids and the flow is weak in the region onshore of the front. Near the nose, the flow turns onshore to fill in this quiescent nearshore region. A scaling for the temporal adjustment to the equilibrated front is proposed. Results from new and previous laboratory experiments spanning two orders of magnitude in $c_{w} / c_{\alpha}$, and previous numerical modelling results all strongly support the scaling theory. Experimentally observed time-dependence of the gravity current width and nose speed is shown to be consistent with viscous interfacial Ekman processes.

We thank Rich Garvine for graciously providing his numerical model results and helpful discussions. We also thank Dave Chapman and Jack Whitehead for helpful discussions. This research was supported by NSF grant OCE-0095059. This is Woods Hole Oceanographic Institution Contribution Number 10575.

\section{REFERENCES}

Avicola, G. \& HuQ, P. 2002 Scaling analysis for the interaction between a buoyant coastal current and the continental shelf: experiments and observations. J. Phys. Oceanogr. (to appear).

BARTH, J. 1989 a Stability of a coastal upwelling front, 1, model development and a stability theorem. J. Phys. Oceanogr. 94(C8), 10844-10856.

BARTH, J. $1989 b$ Stability of a coastal upwelling front, 2, model results and comparison with observations. J. Phys. Oceanogr. 94(C8), 10857-10883.

Chaо, S.-Y. 1988 River-forced estuarine plumes. J. Phys. Oceanogr. 18, 72-88.

Chapman, D. C. \& LentZ, S. J. 1994 Trapping of a coastal density front by the bottom boundary layer. J. Phys. Oceanogr. 24, 1464-1479.

FonG, D. 1998 Dynamics of freshwater plumes: observations and numerical modeling of the wind- 
forced response and alongshore freshwater transport. PhD Thesis, MIT/WHOI Joint Program in Oceanography.

Garvine, R. W. 1999 Penetration of buoyant coastal discharge onto the continental shelf: a numerical model experiment. J. Phys. Oceanogr. 29, 1892-1909.

GARvine, R. W. 2001 Artifacts in buoyant coastal discharge models: an observational and model study. J. Mar. Res. 59, 193-225.

Griffiths, R. W. 1986 Gravity currents in rotating systems. Annu. Rev. Fluid Mech. 18, 59-89.

Griffiths, R. W. \& Linden, P. F. 1981 The stability of buoyancy driven coastal currents. Dyn. Atmos. Oceans 5, 281-306.

Griffiths, R. W. \& Linden, P. F. 1982 Density-driven boundary currents. Geophys. Astrophys. Fluid Dyn. 19, 159-187.

Griffiths, R. W. \& Hopfinger, E. J. 1983 Gravity currents moving along a lateral boundary in a rotating frame. J. Fluid Mech. 134, 357-399.

Helfrich, K. R., Kuo, A. C. \& Pratt, L. J. 1999 Nonlinear Rossby adjustment in a channel. J. Fluid Mech. 390, 187-222.

Hickey, B. M., Pietrafesa, L. J., Jay, D. A. \& W. C. Boicourt, W. C. 1998 The Columbia River Plume Study: subtidal variability in the velocity and salinity fields. J. Geophys. Res. 103(C5), 10339-10368.

Kourafalou, V. H., Oey, L.-Y., Wang, J. D. \& LeE, T. N. 1996 The fate of river discharge on the continental shelf, 1, Modeling the river plume and the inner shelf coastal current. J. Geophys. Res. 101(C2), 3415-3434.

Kubokawa, A. \& K. Hanawa, K. $1984 a$ A theory of semigeostrophic gravity waves and its application to the intrusion of a density current along a coast. Part 1. Semigeostrophic gravity waves. J. Oceanogr. Soc. Japan 40, 247-259.

Kubokawa, A. \& K. Hanawa, K. $1984 b$ A theory of semigeostrophic gravity waves and its application to the intrusion of a density current along a coast. Part 2. Intrusion of a density current along a coast in a rotating fluid. J. Oceanogr. Soc. Japan 40, 260-270.

LeBlond, P. H. \& MysaK, L. A. 1978 Waves in the Ocean. Elsevier.

Munchow, A. \& GARVINE, R. W. 1993 Dynamical properties of a buoyancy-driven coastal current. J. Geophys. Res. 98(C11), 20063-20077.

Pichevin, T. \& NoF, D. 1997 The momentum imbalance paradox. Tellus 49A, 298-319.

Rennie, S., Largier, J. L. \& Lentz, S. J. 1999 Observations of low-salinity coastal current pulses downstream of Chesapeake Bay. J. Geophys. Res. 104(C8), 18227-18240.

ReszKa, M. K. \& Swaters, G. E. 1999 Eddy formation and interaction in a baroclinic frontal geostrophic model. J. Phys. Oceanogr. 29, 3025-3042.

RotTMAn, J. W. \& Simpson, J. E. 1983 Gravity currents produced by instantaneous releases of heavy fluid in a rectangular channel. J. Fluid Mech. 135, 95-110.

Stern, M. E., Whitehead, J. A. \& Hu, B. L. 1982 The intrusion of the head of a gravity current along the coast of a rotating fluid. J. Fluid Mech. 123, 237-266.

Simpson, J. H. 1997 Gravity Currents: in the Environment and the Laboratory, 2nd Edn. Cambridge University Press.

Whitehead, J. A. \& Chapman, D. C. 1986 Laboratory observations of a gravity current on a sloping bottom: the generation of shelf waves. J. Fluid Mech. 172, 373-399.

XING, J. \& Davies, A. M. 1999 The effect of wind direction and mixing upon the spreading of a buoyant plume in a non-tidal regime. Cont. Shelf Res. 19, 1437-1483.

YANkovsky, A. E. \& Chapman, D. C. 1997 A simple theory for the fate of buoyant coastal discharges. J. Phys. Oceanogr. 27, 1386-1401. 